\title{
Generalized Kac-Moody Algebras from CHL dyons
}

\author{
Suresh Govindarajan* \\ Department of Physics, Indian Institute of Technology Madras, \\ Chennai 600036, INDIA. \\ and \\ K. Gopala Krishnađ \\ The Institute of Mathematical Sciences, \\ CIT Campus, Taramani, Chennai 600113 INDIA
}

\begin{abstract}
We provide evidence for the existence of a family of generalized Kac$\operatorname{Moody}(\mathrm{GKM})$ superalgebras, $\mathcal{G}_{N}$, whose Weyl-Kac-Borcherds denominator formula gives rise to a genus-two modular form at level $N, \Delta_{k / 2}(\mathbf{Z})$, for $(N, k)=(1,10),(2,6),(3,4)$, and possibly $(5,2)$. The square of the automorphic form is the modular transform of the generating function of the degeneracy of CHL dyons in asymmetric $\mathbb{Z}_{N}$-orbifolds of the heterotic string compactified on $T^{6}$. The new generalized Kac-Moody superalgebras all arise as different 'automorphic corrections' of the same Lie algebra and are closely related to a generalized Kac-Moody superalgebra constructed by Gritsenko and Nikulin. The automorphic forms, $\Delta_{k / 2}(\mathbf{Z})$, arise as additive lifts of Jacobi forms of (integral) weight $k / 2$ and index $1 / 2$. We note that the orbifolding acts on the imaginary simple roots of the unorbifolded GKM superalgebra, $\mathcal{G}_{1}$ leaving the real simple roots untouched. We anticipate that these superalgebras will play a role in understanding the 'algebra of BPS states' in CHL compactifications.
\end{abstract}

\footnotetext{
*suresh@physics.iitm.ac.in
}

${ }^{\dagger}$ gkrishna@imsc.res.in 


\section{Introduction}

The advent of D-branes and in particular, the successful microscopic description of the entropy of supersymmetric black holes by Strominger and Vafa [1] has provided enormous impetus to the counting of BPS states in a variety of settings. Among these, the $\mathcal{N}=4$ supersymmetric theories, have provided a fairly robust playground for testing and developing new ideas. The high degree of supersymmetry ensures the existence of non-renormalization theorems as well as the existence of dualities. This paper focuses on four-dimensional superstring compactifications, the CHL orbifolds, with $\mathcal{N}=4$ supersymmetry. Such theories typically have a triality of descriptions as heterotic and type IIA/IIB string theories [2].

The spectrum of $1 / 2$-BPS states is independent of moduli - there is no 'jumping' phenomenon. In a remarkable paper [3], Dijkgraaf, Verlinde and Verlinde proposed that the degeneracy of $1 / 4$-BPS states in the heterotic string compactified on $T^{6}$ is generated by a Siegel modular form of weight 10. More precisely, the degeneracy $d(n, \ell, m)$ is given by

$$
\frac{64}{\Phi_{10}(\mathbf{Z})}=\sum_{(n, \ell, m)>0} d(n, \ell, m) q^{n} r^{\ell} s^{m}
$$

where $\mathbf{Z} \in \mathbb{H}_{2}$, the Siegel upper-half space and $(n, \ell, m)=\left(\frac{1}{2} \mathbf{q}_{\mathbf{e}}{ }^{2}, \mathbf{q}_{\mathbf{e}} \cdot \mathbf{q}_{\mathbf{m}}, \frac{1}{2} \mathbf{q}_{\mathbf{m}}{ }^{2}\right)$ are T-duality invariant combinations of electric and magnetic charges. A key feature of the formula is that it is also S-duality invariant i.e., the modular form is invariant under an $S L(2, \mathbb{Z})$ suitably embedded in $S p(2, \mathbb{Z})$. In ref. [4], Gritsenko and Nikulin have shown the existence of a generalized Kac-Moody (GKM) superalgebra, $\mathcal{G}_{1}$, whose Weyl-Kac-Borcherds denominator formula gives rise to a modular form (with character) of weight $5, \Delta_{5}(\mathbf{Z})$, which squares to give $\Phi_{10}(\mathbf{Z})$. It has been anticipated by Harvey and Moore $[5,6]$ that the algebra of BPS states must form an algebra and one suspects that the GKM superalgebra, $\mathcal{G}_{1}$, must play such a role in this example. However, this correspondence has not been fully realised for this example(see ref. [7] however for some recent progress). One of our motivations has been to understand the relation of the algebra of BPS states to this GKM superalgebra.

A family of asymmetric orbifolds of the heterotic string compactified on $T^{6}$ leads to heterotic compactifications that preserve $\mathcal{N}=4$ supersymmetry but the gauge symmetry is of reduced rank [8]. These are called the CHL compactifications. In ref. [9], Jatkar and Sen, constructed a family of genus-two modular forms, $\Phi_{k}(\mathbf{Z})$,

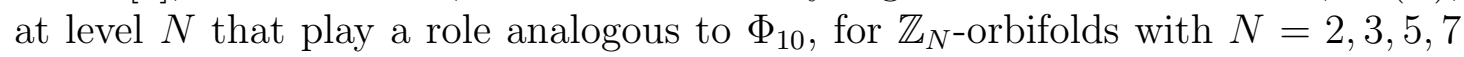

\footnotetext{
${ }^{3}$ We choose a normalization for the modular forms that is natural to the Weyl denominator formula. For instance, our $\Delta_{5}(\mathbf{Z})$ is $1 / 64$ of the same form defined by Gritsenko and Nikulin [4]. Further, $64=2^{\frac{12}{2}}$ also happens to be the size of a $1 / 4$ BPS multiplet of states.
} 
and $(k+2)=24 /(N+1)$. The modular group is a subgroup of $S p(2, \mathbb{Z})$ reflecting the fact that the orbifolding breaks the S-duality group to the sub-group, $\Gamma_{1}(N)$, of $S L(2, \mathbb{Z})[10]$. The main result of this paper is to provide evidence the existence of a family of GKM superalgebras, $\mathcal{G}_{N}$, by showing the existence of modular forms, $\Delta_{k / 2}(\mathbf{Z})$, which: (i) square to $\Phi_{k}(\mathbf{Z})$ and (ii) appear as the denominator formula for $\mathcal{G}_{N}$ for $N=2,3,5$. These constructions parallel the construction of a family of GKM algebras as $\mathbb{Z}_{N}$-orbifolds of the fake Monster Lie algebra [11] by Niemann $[12]$.

We will show that the modular forms $\Delta_{k / 2}(\mathbf{Z})$ are indeed given by the denominator formula for GKM superalgebras, $\mathcal{G}_{N}$, that are closely related to the GKM superalgebra constructed by Gritsenko and Nikulin (we call the superalgebra $\mathcal{G}_{1}$ ) from the modular form $\Delta_{5}(\mathbf{Z})$. In particular, we observe

1. All the algebras arise as (different) automorphic corrections to the Lie algebra $\mathfrak{g}\left(A_{1, I I}\right)$ associated with a rank three Cartan matrix $A_{1, I I}$. In other words, one has $\mathfrak{g}\left(A_{1, I I}\right) \subset \mathcal{G}_{N}$.

2. The real simple roots (and hence the Cartan matrix $A_{1, I I}$ ) for the $\mathcal{G}_{N}$ are identical to the real roots of $\mathfrak{g}\left(A_{1, I I}\right)$. This implies that the Weyl group is identical as well].

3. The multiplicities of the imaginary simple roots are, however, different. For instance, imaginary roots of the form $t \eta_{0}$, where $\eta_{0}$ is a primitive light-like simple root, have a multiplicity $m\left(t \eta_{0}\right)$ given by the formula:

$$
1-\sum_{t \in \mathbb{N}} m\left(t \eta_{0}\right) q^{n}=\prod_{n \in \mathbb{N}}\left(1-q^{n}\right)^{\frac{k-4}{2}}\left(1-q^{N n}\right)^{\frac{k+2}{2}}
$$

Note that this formula correctly reproduces the answer given for $\mathcal{G}_{1}$ by Gritsenko and Nikulin [4].

4. There are also other imaginary simple roots which are not light-like whose multiplicities are determined implicitly by the modular form $\Delta_{k / 2}(\mathbf{Z})$.

The organization of the paper is as follows: After the introductory section, section 2 provides the necessary physical background on CHL strings and the counting of $1 / 4$-BPS dyons in the theory. Section 3 provides the necessary mathematical background leading to the Weyl-Kac-Borcherds denominator formula for GKM algebras. In section 4, which contains the main results of the paper, we provide

\footnotetext{
${ }^{4}$ However, for $N>1$, this Weyl group is no longer the symmetry group of the lattice of dyonic charges as it was for $N=1$. The reason is that the lattice of dyonic charges is not generated by $1 / \Phi_{k}(\mathbf{Z})$. Of course, as was shown by Jatkar and Sen [9], it is another closely related modular form $1 / \widetilde{\Phi}_{k}(\mathbf{Z})$ that generates the lattice of dyonic charges and their degeneracies.
} 
evidence for the existence of Siegel modular forms with character, $\Delta_{k / 2}(\mathbf{Z})$, at level $N$ as the additive lift of weak Jacobi forms of half-integral index. In sections 4.2, 4.3 and 4.4, we show that these modular forms arise as the Weyl-Kac-Borcherds denominator formula for GKM superalgebras, $\mathcal{G}_{N}$, with imaginary simple roots whose multiplicities given by the $\Delta_{k / 2}(\mathbf{Z})$. In section 4.5 , we attempt to provide a physical interpretation for the weak Jacobi forms of half-integral index. We conclude with a summary of our results and a discussion in section 5. Several mathematical appendices have been included. In particular, we review the use of weak Jacobi forms in the construction of Siegel modular forms in appendix B. In appendix C, we discuss the additive lift of Jacobi forms with index $1 / 2$ at level $N$. This generalizes an existing result at level 1 and proves the modularity of the forms $\Delta_{k / 2}(\mathbf{Z})$ that we have constructed. Appendix D gives the explicit Fourier expansion for the modular forms $\Delta_{k / 2}(\mathbf{Z})$ that were used in arriving at the results presented in section 4 .

\section{Background on CHL strings}

The heterotic string compactified on $T^{6}$ and its asymmetric $\mathbb{Z}_{N}$ orbifolds provide us with four-dimensional compactifications with $\mathcal{N}=4$ supersymmetry. Writing $T^{6}$ as $T^{4} \times \widetilde{S}^{1} \times S^{1}$, consider the $\mathbb{Z}_{N}$ orbifold given by the transformation corresponding to a $1 / N$ unit of shift in $\widetilde{S}^{1}$ and a simultaneous $\mathbb{Z}_{N}$ involution of the Narain lattice of signature $(4,20)$ associated with the heterotic string compactified on $T^{4}$. This leads to the CHL compactifications of interest in this paper [9]. The heterotic string compactified on $T^{4} \times \widetilde{S}^{1} \times S^{1}$ is dual to the type IIA string compactified on $K 3 \times \widetilde{S}^{1} \times S^{1}$. The $(4,20)$ lattice gets mapped to $H^{*}(K 3, \mathbb{Z})$ in the type IIA theory and the orbifolding $\mathbb{Z}_{N}$ is a Nikulin involution combined with the $1 / N$ shift of $\widetilde{S}^{1}$.

The low-energy theory consists of the following bosonic fields:

(i) the $\mathcal{N}=4$ supergravity multiplet with the graviton, a complex scalar, $S_{H}$ and six graviphotons; and

(ii) $m=([48 /(N+1)]-2) \mathcal{N}=4$ vector multiplets each containing a gauge field and six scalars.

In terms of the variables that appear in the heterotic description, the bosonic part of the low-energy effective action (up to two derivatives) is $[13,15,16]$

$$
\begin{aligned}
S=\int d^{4} x \sqrt{-g}[R- & \frac{\partial_{\mu} S_{H} \partial^{\mu} \bar{S}_{H}}{2 \operatorname{Im}\left(S_{H}\right)^{2}}+\frac{1}{8} \operatorname{Tr}\left(\partial_{\mu} M L \partial^{\mu} M L\right) \\
& \left.-\frac{1}{4} \operatorname{Im}\left(S_{H}\right) F_{\mu \nu} L M L F^{\mu \nu}+\frac{1}{4} \operatorname{Re}\left(S_{H}\right) F_{\mu \nu} L \widetilde{F}^{\mu \nu}\right],
\end{aligned}
$$


where $L$ is a Lorentzian metric with signature $(6, m), M$ is a $(6+m) \times(6+m)$ matrix valued scalar field satisfying $M^{T}=M$ and $M^{T} L M=L$ and $F_{\mu \nu}$ is a $(6+m)$ dimensional vector field strength of the $(6+m)$ gauge fields.

The moduli space of the scalars is given by

$$
\left(\Gamma_{1}(N) \times S O(6, m ; \mathbb{Z})\right) \backslash\left(\frac{S L(2)}{U(1)} \times \frac{S O(6, m)}{S O(6) \times S O(m)}\right)
$$

$S O(6, m ; \mathbb{Z})$ is the T-duality symmetry and $\Gamma_{1}(N) \subset S L(2, \mathbb{Z})$ is the S-duality symmetry that is manifest in the equations of motion and is compatible with the charge quantization [10]. The fields that appear at low-energy can be organized into multiplets of these various symmetries.

1. The heterotic dilaton combines with the axion (obtained by dualizing the antisymmetric tensor) to form the complex scalar $S_{H}$. Under $S$-duality, $S_{H} \rightarrow\left(a S_{H}+b\right) /\left(c S_{H}+d\right)$.

2. The $(6+m)$ vector fields transform as a $S O(6, m ; \mathbb{Z})$ vector. Thus, the electric charges $\mathbf{q}_{e}$ (resp. magnetic charges $\mathbf{q}_{m}$ ) associated with these vector fields are also vectors (resp. co-vectors) of $S O(6, m, \mathbb{Z})$. Further, the electric and magnetic charges 5 transform as a doublet under the $S$-duality group, $\Gamma_{1}(N)$, where $\Gamma_{1}(N)$ is the sub-group of $S L(2, \mathbb{Z})$ matrices $\left(\begin{array}{ll}a & b \\ c & d\end{array}\right)$ with $a=d=1$ $\bmod N$ and $c=0 \bmod N$ ).

One can form three T-duality invariant scalars, $\mathbf{q}_{e}^{2}, \mathbf{q}_{m}^{2}$ and $\mathbf{q}_{e} \cdot \mathbf{q}_{m}$ from the charge vectors. These transform as a triplet of the S-duality group. Equivalently, we can write the triplet as a symmetric matrix:

$$
\mathcal{Q} \equiv\left(\begin{array}{cc}
\mathbf{q}_{e}^{2} & -\mathbf{q}_{e} \cdot \mathbf{q}_{m} \\
-\mathbf{q}_{e} \cdot \mathbf{q}_{m} & \mathbf{q}_{m}^{2}
\end{array}\right)
$$

The $S$-duality transformation now is $\mathcal{Q} \rightarrow A \cdot \mathcal{Q} \cdot A^{T}$ with $A=\left(\begin{array}{ll}a & b \\ c & d\end{array}\right) \in \Gamma_{1}(N)$. The charges are quantized such that $N \mathbf{q}_{e}^{2}, \mathbf{q}_{m}^{2} \in 2 \mathbb{Z}$ and $\mathbf{q}_{e} \cdot \mathbf{q}_{m} \in \mathbb{Z}$. There exist many more invariants due to the discrete nature of the T-duality group [14] for $N=1$ and more appear when $N>1$.

\subsection{BPS multiplets}

Four-dimensional compactifications with $\mathcal{N}=4$ supersymmetry admit two kinds of BPS states: (i) 1/2-BPS multiplets that preserve eight supercharges (with 16 states

\footnotetext{
${ }^{5}$ To be precise, $\mathbf{q}_{e}$ and $-L \mathbf{q}_{m}$ form the doublet. Also note that the Lorentzian inner product is $\mathbf{q}_{e}^{2}=\mathbf{q}_{e}^{T} L \mathbf{q}_{e}$. We shall not indicate the appearance of $L$ in subsequent formulae that appear in the paper.
} 
in a multiplet) and (ii) 1/4-BPS multiplets that preserve four supercharges(with 64 states in a multiplet). The masses of the 1/4-BPS states are determined in terms of their charges by means of the BPS formula $[2,15,16]$ :

$$
\begin{aligned}
\left(M_{ \pm}^{2}\right)_{1 / 4-B P S} & =\frac{1}{S_{H}-\bar{S}_{H}}\left[\left(\mathbf{q}_{e}+S_{H} \mathbf{q}_{m}\right)^{T}(M+L)\left(\mathbf{q}_{e}+\bar{S}_{H} \mathbf{q}_{m}\right)\right. \\
\pm \frac{1}{2} & \left.\sqrt{\left(\mathbf{q}_{e}^{T}(M+L) \mathbf{q}_{e}\right)\left(\mathbf{q}_{m}^{T}(M+L) \mathbf{q}_{m}\right)-\left(\mathbf{q}_{e}^{T}(M+L) \mathbf{q}_{m}\right)^{2}}\right]
\end{aligned}
$$

The square of the mass of a $1 / 4$-BPS state is $\max \left(M_{+}^{2}, M_{-}^{2}\right)$. 1/2-BPS states appear when the electric and magnetic charges are parallel (or anti-parallel) i.e., $\mathbf{q}_{e} \propto L \mathbf{q}_{m}$. The BPS mass formula for $1 / 2$-BPS states can be obtained as a specialization of the 1/4-BPS mass formula given above. When $\mathbf{q}_{e} \propto L \mathbf{q}_{m}$, the terms inside the square root appearing in the 1/4-BPS mass formula vanish leading to the $1 / 2$-BPS formula

$$
\left(M^{2}\right)_{1 / 2-B P S}=\frac{1}{S_{H}-\bar{S}_{H}}\left[\left(\mathbf{q}_{e}+S_{H} \mathbf{q}_{m}\right)^{T}(M+L)\left(\mathbf{q}_{e}+\bar{S}_{H} \mathbf{q}_{m}\right)\right]
$$

\subsection{Counting $1 / 2$-BPS states}

We will now consider the counting of purely electrically charged $1 / 2$-BPS states. Such electrically charged states are in one to one correspondence with the states of the CHL orbifold of the heterotic string compactified on $T^{4} \times T^{2}$ [10]. Let $d(n)$ denote the degeneracy of heterotic string states carrying charge $N \mathbf{q}_{e}^{2}=2 n$ - the fractional charges arise from the twisted sectors in the CHL orbifolding. Every $1 / 2$-BPS multiplet/heterotic string state has degeneracy $16=2^{8 / 2}$. Then the generating function of $d(n)$ is [17-19]

$$
\sum_{n=0}^{\infty} d(n) \exp \left(\frac{2 \pi i n \tau}{N}\right)=\frac{16}{(i \sqrt{N})^{-k-2} f^{(k)}(\tau / N)}
$$

where

$$
f^{(k)}(\tau) \equiv \eta(N \tau)^{k+2} \eta(\tau)^{k+2} .
$$

The degeneracy of purely magnetically charged states with charge $\mathbf{q}_{m}=2 \mathrm{~m}$ is given by a similar formula with $f^{(k)}(\tau / N)$ replaced by $f^{(k)}(\tau)$. These are level- $N$ modular forms with weight $(k+2)$. For $(N, k)=(1,10), f^{(10)}(\tau)=\eta(\tau)^{24}$.

\subsection{Counting $1 / 4$-BPS states}

As we saw earlier, 1/4-BPS states are necessarily dyonic in character with the electric and magnetic charge vectors being linearly independent. In a remarkable 
leap of intuition, Dijkgraaf, Verlinde and Verlinde (DVV) proposed that a Siegel modular form of weight 10 is the generating function of $1 / 4$-BPS states [3]. Let $d(n, \ell, m)$ be the degeneracy of $1 / 4$-BPS states with charges $\mathbf{q}_{e}^{2}=2 n, \mathbf{q}_{m}^{2}=2 m$ and $\mathbf{q}_{e} \cdot \mathbf{q}_{m}=\ell$. Then, one has

$$
\sum_{(n, \ell, m)>0} d(n, \ell, m) q^{n} r^{\ell} s^{m}=\frac{64}{\Phi_{10}(\mathbf{Z})},
$$

with $\mathbf{Z}=\left(\begin{array}{cc}z_{1} & z_{2} \\ z_{2} & z_{3}\end{array}\right) \in \mathbb{H}_{2}$ and $q=\exp \left(2 \pi i z_{1}\right), r=\exp \left(2 \pi i z_{2}\right), s=\exp \left(2 \pi i z_{3}\right)$ (see Appendix (B.1) for further details) and $(n, \ell, m)>0$ implies $n, m \geq 1, \ell \in \mathbb{Z}$ and $\left(4 n m-\ell^{2}\right)>0$. The modular form $\Phi_{10}(\mathbf{Z})$ has a double zero at $z_{2}=0$, where it factorizes as

$$
\lim _{z_{2} \rightarrow 0} \Phi_{10}(\mathbf{Z})=\left(2 \pi z_{2}\right)^{2} \eta\left(z_{1}\right)^{24} \eta\left(z_{3}\right)^{24}
$$

In this limit, one sees the appearance of the degeneracies of the pure electric and magnetic states - these are generated by $\eta\left(z_{1}\right)^{24}$ and $\eta\left(z_{3}\right)^{24}$ respectively. From this we see that the $\mathbb{Z}_{2}$ transformation which exchanges electric and magnetic charges corresponds to the geometric action on $\mathbb{H}_{2}: z_{1} \leftrightarrow z_{3}$. Another check of this formula is that this modular form (as well as the ones that we discuss later) matches the Bekenstein-Hawking entropy of large dyonic blackholes in the limit of large electric and magnetic charges 6 .

In a development that has spurred activity in this area, Jatkar and Sen generalized the DVV proposal to the case of asymmetric $\mathbb{Z}_{N}$-orbifolds of the heterotic string on $T^{6}$ for $N=2,3,5,7[9]$. They proposed that the degeneracy of $1 / 4$-BPS dyons is generated by a Siegel modular form of weight $k=\frac{24}{N+1}-2$ and level $N$, $\widetilde{\Phi}_{k}(\mathbf{Z})$. They also provided an explicit construction of the modular form using the additive lift of a weak Jacobi form. The constructed modular form has the following properties:

(i) It is invariant under the S-duality group $\Gamma_{1}(N)$ suitably embedded in the group $G_{1}(N) \subset S p(2, \mathbb{Z})$.

(ii) In the limit $z_{2} \rightarrow 0$, it has the right factorization property:

$$
\lim _{z_{2} \rightarrow 0} \widetilde{\Phi}_{k}(\mathbf{Z})=(i \sqrt{N})^{-k-2}\left(2 \pi z_{2}\right)^{2} f^{(k)}\left(z_{1} / N\right) f^{(k)}\left(z_{3}\right)
$$

Note that for $(N, k)=(1,10)$, this matches the DVV formula.

(iii) It reproduces the entropy for large blackholes [9].

\footnotetext{
${ }^{6} \mathrm{~A}$ more precise statement is that the formula reproduces the entropy of large blackholes with torsion one. The degeneracy of $1 / 2$-BPS blackholes is more complicated to understand as they have no horizon and hence have vanishing Bekenstein-Hawking entropy.
} 
(iv) For 1/2-BPS blackholes, the formula leads to a prediction for $R^{2}$ (higher derivative) corrections to the low-energy effective action given in Eq. (2.1). Such corrections lead to a non-zero entropy using Wald's generalization of the BH entropy formula for Einstein gravity that agrees with the prediction from the modular form $[20,21]$.

The S-duality group $\Gamma_{1}(N)$ as well as $\Gamma_{0}(N) \in S L(2, \mathbb{Z})$ are embedded into $S p(2, \mathbb{Z})$ as follow: 7 :(Note that this is not quite the embedding given by Jatkar-Sen - we have carried out a $z_{1} \leftrightarrow z_{3}$ exchange on their embedding to match our notation.)

$$
\left(\begin{array}{ll}
a & b \\
c & d
\end{array}\right) \mapsto\left(\begin{array}{cccc}
d & -c & c & 0 \\
-b & a & 0 & b \\
0 & 0 & a & b \\
0 & 0 & c & d
\end{array}\right), \quad c=0 \quad \bmod N
$$

with the additional condition $a=1 \bmod N$ for $\Gamma_{1}(N)$. Let us call this sub-group of $S p(2, \mathbb{Z}), G_{0}(N)$. Further, let $\Gamma^{J}(N) \equiv \Gamma^{J} \cap G_{0}(N)$. This is subgroup of $G_{0}(N)$ that preserves the cusp at $\left(z_{3}\right)=i \infty$ (see appendix B). The weak Jacobi form $\phi_{k, 1}\left(z_{1}, z_{2}\right)$ that generates $\tilde{\Phi}_{k}(\mathbf{Z})$ is a modular form of $\Gamma^{J}(N)$. Then, for prime $N$, there are two inequivalent cusps in the upper-half plane $\mathbb{H}_{1}$ corresponding to $\left(z_{1}\right)=0$ and $\left(z_{1}\right)=i \infty$. Thus, the modular form $\tilde{\Phi}_{k}(\mathbf{Z})$ behaves differently at the two cusps. Its behavior at $z_{1}=0$ is captured by the modular form $\Phi_{k}(\mathbf{Z})$ also defined by Jatkar and Sen [9]. One has

$$
\Phi_{k}(\mathbf{Z}) \equiv z_{1}^{-k} \widetilde{\Phi}_{k}(\widetilde{\mathbf{Z}})
$$

with

$$
\tilde{z}_{1}=-1 / z_{1} \quad, \quad \tilde{z}_{2}=z_{2} / z_{1} \quad, \quad \tilde{z}_{3}=z_{3}-z_{2}^{2} / z_{1} .
$$

It is not hard to see that the above change of variables maps $\left(z_{1}\right)=0$ to $\left(z_{1}\right)=i \infty$ while preserving the cusp at $\left(z_{3}\right)=i \infty$. We will be dealing with the modular form, $\Phi_{k}(\mathbf{Z})$, for most of this paper as it is symmetric in the 'electric' and 'magnetic' variables $z_{1}$ and $z_{3}$ even though it is $\widetilde{\Phi}_{k}(\mathbf{Z})$ which is the generating function of dyonic degeneracies. Note that this point is not relevant for $N=1$ (c.f. the DVV formula) as both the cusps get identified under $S L(2, \mathbb{Z})$.

\subsection{Product formulae for the modular forms}

Product representations for a closely related modular form $\Phi_{k}(\mathbf{Z})$ were provided by two groups $[17,22]$. In a subsequent paper [23], David and Sen derived the

\footnotetext{
${ }^{7}$ It turns out that the modular forms constructed by Jatkar-Sen are invariant under the larger group $\Gamma_{0}(N)$ for $N=2,3,5$.
} 
product representations for modular form $\tilde{\Phi}_{k}(\mathbf{Z})$ as well as $\Phi_{k}(\mathbf{Z})$ using the $4 \mathrm{D}-5 \mathrm{D}$ correspondence with the well-studied $D 1-D 5$ system in Taub-NUT space [24]. They showed that it can be written as the product of three contributions arising from: (i) the low-energy dynamics in Taub-NUT space, (ii) the center of mass dynamics of the D1-D5 system in Taub-NUT space and (iii) the dynamics of D1branes along $K 3 / \mathbb{Z}_{N} 8$.

$$
\widetilde{\Phi}_{k}(\mathbf{Z})=(i \sqrt{N})^{-k-2} f_{k}\left(z_{1} / N\right) \times \mathcal{E}_{\mathrm{st} \times T^{2}}\left(z_{1}, z_{2}\right) \times \hat{\mathcal{E}}_{S^{*}\left(K 3 / \mathbb{Z}_{N}\right)}\left(z_{1}, z_{2}, z_{3}\right)
$$

where $\mathcal{E}_{\text {st } \times T^{2}}\left(z_{1}, z_{2}\right)=\left[\vartheta_{1}\left(z_{1}, z_{2}\right) / \eta\left(z_{1}\right)^{3}\right]^{2}$ is the spacetime elliptic genus [16] and $\hat{\mathcal{E}}_{S^{*}\left(K 3 / \mathbb{Z}_{N}\right)}\left(z_{1}, z_{2}, z_{3}\right)$ is the 'second-quantized elliptic genus' of $K 3 / \mathbb{Z}_{N}$ defined by $[23,25]$

$$
\hat{\mathcal{E}}_{S^{*}\left(K 3 / \mathbb{Z}_{N}\right)}\left(z_{1}, z_{2}, z_{3}\right)=\sum_{p=0}^{\infty} \mathcal{E}_{S^{p}\left(K 3 / \mathbb{Z}_{N}\right)}\left(z_{1}, z_{2}\right) e^{2 \pi i p z_{3}}
$$

where $\mathcal{E}_{S^{p}\left(K 3 / \mathbb{Z}_{N}\right)}$ is the elliptic genus of the $p$-th symmetric product of $K 3 / \mathbb{Z}_{N}$ and $\mathcal{E}_{S^{0}\left(K 3 / \mathbb{Z}_{N}\right)} \equiv 1$. Note that each of the terms in Eq. (2.12) independently admits a product representation - for details see [23].

We will obtain product representations for $\Phi_{k}(\mathbf{Z})$ for $N=2,3,5$ as the multiplicative (Borcherds) lift of a Jacobi form $\phi_{0,1}^{(N)}(\tau, z)$ of $\Gamma_{0}(N)^{J}=\Gamma_{0}(N) \ltimes \mathbb{Z}^{2}[35]$. For the cases of interest, the groups have two cusps at $\tau=i \infty$ and $\tau=0$. Let $c_{1}(n, \ell)$ and $c_{2}(n, \ell)$ be the coefficients of the Fourier expansion about the two cusps. Then, one has $[22,35]$

$$
\Phi_{k}(\mathbf{Z})=q r s \prod_{n, \ell, m \in \mathbb{Z}}\left(1-q^{n} r^{\ell} s^{m}\right)^{c_{1}(n m, \ell)} \times \prod_{n, \ell, m \in \mathbb{Z}}\left(1-\left(q^{n} r^{\ell} s^{m}\right)^{N}\right)^{c_{2}(n m, \ell)}
$$

Note that we use a normalization for $\Phi_{k}(\mathbf{Z})$ that differs by on an overall sign from the one used by David-Jatkar-Sen [17]. To make use of this formula, we need to determine the relevant Jacobi form. According to Aoki-Ibukiyama (Prop. 6.1 in [35]), any weak Jacobi form of weight zero and index one of $\Gamma_{0}(N)^{J}$ is of the form

$$
\phi_{0,1}^{(N)}(\tau, z)=A^{(N)} \alpha^{(N)}(\tau) \phi_{-2,1}(\tau, z)+B^{(N)} \phi_{0,1}(\tau, z),
$$

where $\phi_{-2,1}(\tau, z)$ and $\phi_{0,1}(\tau, z)$ are as defined in Eq. (B.9) and $\alpha^{(N)}(\tau)$ is a weight two modular form at level $N$ and $A^{(N)}$ and $B^{(N)}$ are constants. When $N=2,3,5$, there is only one such weight two modular form with constant coefficient $=1$ given by the Eisenstein series: $\alpha^{(N)}(\tau)=\frac{12 i}{\pi(N-1)} \partial_{\tau}[\ln \eta(\tau)-\ln \eta(N \tau)]$. Thus, our problem is reduced to fixing two constant coefficients, $A^{(N)}$ and $B^{(N)}$ which we do now following the procedure given in ref. [22].

\footnotetext{
${ }^{8}$ The $\mathbb{Z}_{N}$ is the Nikulin involution associated with the type IIA dual of the CHL orbifold.
} 
Recall that $\phi_{k, 1}(\tau, z)$, that generates the additive lift for $\Phi_{k}(\mathbf{Z})$, provides all the terms in $\Phi_{k}(\mathbf{Z})$ that appear with coefficient $s[9]$. The product representation of the Jacobi form, $\phi_{k, 1}(\tau, z)$, thus enables us to fix the coefficients in Eq. (2.13) with $m=0$. Comparing the product form (referred to as the Hodge anomaly in [22]) given below with Eq. (2.13)

$$
\begin{aligned}
& \phi_{k, 1}(\tau, z)=\eta(\tau)^{k-2} \eta(N \tau)^{k+2} \vartheta_{1}^{2}(\tau, z), \\
& \quad=q r\left(1-r^{-1}\right)^{2} \prod_{n=1}^{\infty}\left(1-q^{n}\right)^{k-2}\left(1-q^{n N}\right)^{k+2}\left(1-q^{n} r\right)^{2}\left(1-q^{n} r^{-1}\right)^{2},
\end{aligned}
$$

we see that

$$
c_{1}(-1)=2, \quad c_{1}(0)=(k-2), \quad c_{2}(-1)=0 \text { and } \quad c_{2}(0)=(k+2),
$$

where the argument of $c_{s}$ is given in terms of $\left(4 n m-\ell^{2}\right)$. Note that $(k+2)=$ $24 /(N+1)$ as usual. For instance, the coefficient of $r$ at the cusp at $i \infty$ implies $A^{(N)}+\left(B^{(N)} / 2\right)=c_{1}(-1)$ and similarly, the constant term at the same cusp implies $-2 A^{(N)}+5 B^{(N)}=c_{1}(0)$. This implies that

$$
A^{(N)}=\frac{2 N}{N+1} \quad, \quad B^{(N)}=\frac{1}{N+1},
$$

which match the results given in ref. [22] for $N=2$. These two conditions did not need too much detail about the weight two modular form $\alpha^{(N)}(\tau)$ other than its normalization, i.e., $\alpha^{(N)}(i \infty)=1$. The expansion at the other cusp provide an infinite number of consistency checks of the existence of the multiplicative lift.

The product expansion for $\Phi_{k}(\mathbf{Z})$ that has been given by David-Jatkar-Sen in [17] naively appears to be of a different form. However, one can show that the two expansions are indeed the same providing us with an additional check on our product formula 9 Further, it gives an alternate formula for $c_{2}(n, \ell)$ as the Fourier coefficients of another weak Jacobi form

$$
\widehat{\phi}_{0,1}^{(N)}(\tau, z)=\frac{-2}{N+1} \alpha^{(N)}(\tau) \phi_{-2,1}(\tau, z)+\frac{1}{N+1} \phi_{0,1}(\tau, z)
$$

about the cusp at $i \infty$. It is also important, for our later discussion, to note that the coefficients $c_{1}(n, \ell)$ and $c_{2}(n, \ell)$ are all even integers for $N=2,3,5$ to the orders $(n \leq 12)$ that we have checked. We believe that this is true to all orders.

\footnotetext{
${ }^{9}$ We thank Justin David for useful discussions and in particular, for informing us about this relationship.
} 


\section{Generalized Kac-Moody algebras}

\subsection{From Cartan Matrices to Lie Algebras}

A Lie algebra is defined as a vector space $\mathfrak{g}$ with an anti-symmetric bilinear map $[]:, \mathfrak{g} \times \mathfrak{g} \mapsto \mathfrak{g}$ satisfying the Jacobi identity. A finite dimensional Lie algebra can also be defined through its Cartan matrix. Given a real, indecomposable, $(r \times r)$ symmetric matrix $A=\left(a_{i j}\right), i, j \in I=\{1,2, \ldots, r\}$ of rank $r$ satisfying the following conditions:

(i) $a_{i i}=2$,

(ii) $a_{i j}=0 \Leftrightarrow a_{j i}=0$,

(iii) $a_{i j} \in \mathbb{Z}_{\leq 0}$ for $i \neq j$,

(iv) $\operatorname{det} A>0$,

one defines a Lie algebra $\mathfrak{g}(A)$ generated by the generators $e_{i}, f_{i}$, and $h_{i}$, for $i \in I$, satisfying the following conditions for $i, j \in I$ :

$$
\begin{aligned}
& {\left[h_{i}, h_{j}\right]=0 ;\left[e_{i}, f_{j}\right]=\delta_{i j} h_{j} ;\left[h_{i}, e_{j}\right]=a_{i j} e_{j} ;\left[h_{i}, f_{j}\right]=-a_{i j} f_{j}} \\
& \quad\left(\operatorname{ad} e_{i}\right)^{1-a_{i j}} e_{j}=\left(\operatorname{ad} f_{i}\right)^{1-a_{i j}} f_{j}=0 \text { for } i \neq j .
\end{aligned}
$$

The Cartan matrix thus uniquely defines the Lie algebra which we call $\mathfrak{g}(A)$ (and on occasion simply $A$ ).

More general classes of Lie algebras are obtained by weakening the axioms on the Cartan matrix. In particular, relaxing condition (iv) on the positive definiteness of the determinant of the Cartan matrix yields Kac-Moody algebras which are infinite dimensional. Relaxing conditions (i) and (iv), one obtains the most general class of Kac-Moody algebras, the generalized Kac-Moody (GKM) algebras.

Affine Lie algebras are obtained by requiring positive semi-definiteness of the determinant of $A$ in the place of positive definiteness. Thus, for an affine Lie algebra the condition on the determinant is,

$$
\operatorname{det} A_{\{i\}}>0
$$

for all $i=1, \ldots, r$, where $A_{\{i\}}$ are the matrices obtained from A by deleting the $i$-th row and column. For an affine Lie algebra, the rank of $A$ is at least $(r-1)$.

\footnotetext{
${ }^{10}$ The symmetric condition can be extended to include symmetrizable matrices. A matrix $A$ is said to be symmetrizable if there exists a non-degenerate diagonal matrix $D$ such that $A=D B$ where $B$ is a symmetric matrix.
} 
Our main focus will be on the following sequence of Cartan matrices

$$
A_{1}=(2) \hookrightarrow A_{1}^{(1)}=\left(\begin{array}{rr}
2 & -2 \\
-2 & 2
\end{array}\right) \hookrightarrow A_{1, I I} \equiv\left(\begin{array}{rrr}
2 & -2 & -2 \\
-2 & 2 & -2 \\
-2 & -2 & 2
\end{array}\right)
$$

The first matrix in the sequence leads to the finite rank one Lie algebra $A_{1}$, the second leads to the affine Lie algebra $\hat{A}_{1}^{(1)}$. The Lie algebra $\mathfrak{g}\left(A_{1, I I}\right)$ is a sub-algebra of the GKM superalgebras $11, \mathcal{G}_{N}$, that we construct in this paper.

\subsection{Cartan subalgebra}

The $\mathbb{Z}$-span $\mathfrak{h}$, of the elements $\left\{h_{i}\right\}$ is called the Cartan subalgebra of $\mathfrak{g}(A)$. The Lie algebra decomposes into eigenspaces, called root spaces, under the simultaneous adjoint action of $\mathfrak{h}$. An element $\alpha \in \mathfrak{h}^{*}$ is called a (real) root if the eigenspace

$$
\mathfrak{g}_{\alpha}=\{g \in \mathfrak{g} \mid[h, g]=\alpha(h) g, \forall h \in \mathfrak{h}\}
$$

defined by $\alpha$ is not empty. The set $L$, of all $\alpha$ such that $\mathfrak{g}_{\alpha} \neq 0$, is called the root system. The root space is generated by elements $\alpha_{i}$ satisfying

$$
\alpha_{i}\left(h_{j}\right)=a_{i j} \quad \text { for } \quad i, j \in I .
$$

The elements $\alpha_{i}$ are called the simple roots and the set of roots are generated as integral linear combinations of these with coefficients either all positive, or all negative. These sets are called the set of positive and negative roots respectively, and are denoted by $L_{+}$and $L_{-}$. Thus, $L=L_{+} \cup L_{-}$. This decomposition gives a grading on the Lie algebra

$$
\mathfrak{g}=\bigoplus_{\alpha \in L} \mathfrak{g}_{\alpha}=\bigoplus_{\alpha \in L_{-}} \mathfrak{g}_{\alpha} \bigoplus \mathfrak{h} \bigoplus_{\alpha \in L_{+}} \mathfrak{g}_{\alpha}
$$

The affine Lie algebras are obtained by 'adding' a root $\alpha_{0}$ to a finite Lie algebra. As the Cartan matrix of an affine Lie algebra is degenerate, there is an element, $k \in \mathfrak{h}$, that is central, i.e., it commutes with all elements of the Lie algebra. For $\hat{A}_{1}^{(1)}$, one has $k=h_{0}+h_{1}$. The non-degeneracy of the Cartan matrix is fixed by adding a new generator to $\mathfrak{h}$ called the derivation, $d$, to the Lie algebra $\mathfrak{g}\left(A_{1}^{(1)}\right)$ with the following Lie brackets [26]:

$$
\left[\alpha_{i}, d\right]=0,\left[\alpha_{0}, d\right]=+\alpha_{0},\left[h_{i}, d\right]=0,[k, d]=0 .
$$

\footnotetext{
${ }^{11} \mathrm{~A}$ superalgebra is an algebra with a $\mathbb{Z}_{2}$ grading - the algebra has bosonic and fermionic elements satisfying a graded Lie bracket. The required generalizations have been discussed in [4] and we refer the reader to it.
} 
The roots are defined on $\mathfrak{h}^{*}$, consequently, modifying $\mathfrak{h}$ implies the root space also gets modified accordingly. The set of positive roots, $L_{+}$, in this case are defined to be the union of the set of positive roots of the finite-dimensional Lie algebra and the set of roots for which

$$
[\alpha, d]=c \alpha, \text { with the constant } c>0 .
$$

For the affine Kac-Moody algebra, $\widehat{A}_{1}^{(1)}$, from the above definition of the set of positive roots, we have

$$
L_{+}=\left(n\left(\alpha_{1}+\alpha_{0}\right), n \alpha_{1}+(n-1) \alpha_{0},(n-1) \alpha_{1}+n \alpha_{0} \mid n=1,2,3, \ldots\right),
$$

and $L_{-}=-L_{+}$.

The class of infinite dimensional algebras that are obtained from a generalized Cartan matrix are known as generalized Kac-Moody algebras have a more general root structure. In general there is no simple way to characterize the generalization from affine to the generalized class of Kac-Moody algebras, as there are many classes of GKM algebras that can be constructed due to the high degree of arbitrariness in the characterization of their Cartan matrices. The root systems are also considerably different from the affine case and can have imaginary simple roots in addition to real simple roots, and the multiplicity of the roots can be hard to determine.

The GKM algebra $\mathfrak{g}\left(A_{1, I I}\right)$ is one which contains $\hat{A}_{1}^{(1)}$ as a sub-algebra and falls into a class of GKM algebras called hyperbolic Kac-Moody algebras that have been classified. There are infinitely many hyperbolic Kac-Moody algebras at rank two. At rank greater than two but less than eleven, there exist a finite number of hyperbolic Kac-Moody algebras. There are no hyperbolic Kac-Moody algebras at rank higher than ten. The embedding of $\hat{A}_{1}^{(1)}$ has been used by Feingold and Frenkel to study another rank three hyperbolic Kac-Moody algebra [27]. More recently, Feingold and Nicolai have shown that this hyperbolic Kac-Moody algebra contains $\mathfrak{g}\left(A_{1, I I}\right)$ as a sub-algebra [28].

\subsection{Weyl groups}

The Weyl group of a Cartan matrix, $\mathcal{W}(A)$, is defined as the group generated by elementary reflections associated with the real simple roots

$$
w_{\alpha_{i}}(\beta)=\beta-2 \frac{\left(\beta, \alpha_{i}\right)}{\left(\alpha_{i}, \alpha_{i}\right)} \alpha_{i}, \quad i \in I
$$

and $\beta$ any root. It is easy to see that $w_{\alpha_{i}}^{2}=1, \forall i \in I$. Further, the Cartan matrix, $A$, determines further relations, if any, amongst the basic reflections and thus these 
groups are Coxeter groups. For symmetric matrices $A$, one has

$$
\left(w_{\alpha_{i}} w_{\alpha_{j}}\right)^{2+\left|a_{i j}\right|^{2}}=1 \text { when }\left|a_{i j}\right|=0,1 \text { and } i \neq j \text {. }
$$

Further, when $\left|a_{i j}\right| \geq 2$, there are no relations.

For the three Cartan matrices given in Eq. (3.2), the Weyl group is generated by $r$ elementary reflections, $w_{\alpha_{i}}$ with no further relations. For $r=1$, this group is $\mathbb{Z}_{2}$. For $r=2,3$, these groups are infinite dimensional. For $r=2$, the affine Weyl group is the semi-direct product $\left(\mathbb{Z}_{2} \ltimes \mathbb{Z}\right)$, where $\mathbb{Z}$ is the normal sub-group of translations generated by $t \equiv\left(w_{\alpha_{1}} w_{\alpha_{2}}\right)$.

\subsubsection{The Weyl group $\mathcal{W}\left(A_{1, I I}\right)$}

We now discuss the Weyl group associated with the Cartan matrix $A_{1, I I}$ given in (3.2) in some detail as it plays an important role in this paper. Let $\left(\delta_{1}, \delta_{2}, \delta_{3}\right)$ be the three real simple roots whose Gram matrix (matrix of inner products) is given by the matrix $A_{1, I I}$. Let $\left(w_{1}, w_{2}, w_{3}\right)$ denote the three reflections generated by the three simple roots. The Weyl group, $\mathcal{W}\left(A_{1, I I}\right)$, is generated by these three elementary reflections with no further relations. It turns out that $\mathcal{W}\left(A_{1, I I}\right)$ has a nicer presentation as a normal subgroup of $P G L(2, \mathbb{Z})$ which we describe later. One has [4] (see also [7,28])

$$
P G L(2, \mathbb{Z})=\mathcal{W}\left(A_{1, I I}\right) \rtimes S_{3},
$$

where $S_{3}$ is the group of permutations of the three real simple roots.

The three real simple roots define the root lattice $M_{I I}=\mathbb{Z} \delta_{1} \oplus \mathbb{Z} \delta_{2} \oplus \mathbb{Z} \delta_{3}$ and a fundamental polyhedron, $\mathcal{M}_{I I}$, which is given by the region bounded by the spaces orthogonal to the real simple roots.

$$
\mathbb{R}_{+} \mathcal{M}_{I I}=\left\{x \in M_{I I} \otimes \mathbb{R} \mid\left(x, \delta_{i}\right) \leq 0, i=1,2,3\right\} .
$$

The lattice $M_{I I}$ has a lattice Weyl vector which is an element $\rho \in M_{I I} \otimes \mathbb{Q}$ such that all the real simple roots satisfy 12

$$
\left(\rho, \delta_{i}\right)=-\frac{\left(\delta_{i}, \delta_{i}\right)}{2}=-1
$$

One has $\rho=\left(\delta_{1}+\delta_{2}+\delta_{3}\right) / 2$ i.e., it is one-half of the sum over real simple roots. The positive real roots are then given by

$$
L_{+}^{\mathrm{re}}=\left(\mathcal{W}\left(\delta_{1}, \delta_{2}, \delta_{3}\right) \cap M_{I I}^{+}\right),
$$

\footnotetext{
${ }^{12}$ The standard convention is to define $\rho$ through the condition $\left(\rho, \delta_{i}\right)=\left(\delta_{i}, \delta_{i}\right) / 2$ for all real simple roots $\delta_{i}$. However, in this subsection, we reproduce the notation of Gritsenko and Nikulin (which differs by a sign) here as we wish to compare with their results in the later part of the paper.
} 
where $\mathcal{W}$ refers to the Weyl group $\mathcal{W}\left(A_{1, I I}\right)$ and $M_{I I}^{+}=\mathbb{Z}_{+} \delta_{1} \oplus \mathbb{Z}_{+} \delta_{2} \oplus \mathbb{Z}_{+} \delta_{3}$.

In order to make the map to $P G L(2, \mathbb{Z})$ explicit, we will choose to write the roots in terms of a basis $\left(f_{2}, f_{3}, f_{-2}\right)$ which are related to the $\delta_{i}$ in the following way:

$$
\delta_{1}=2 f_{2}-f_{3}, \quad \delta_{2}=f_{3}, \quad \delta_{3}=2 f_{-2}-f_{3} .
$$

The non-vanishing inner products among the elements $f_{i}$ are:

$$
\left(f_{2}, f_{-2}\right)=-1, \quad\left(f_{3}, f_{3}\right)=2 .
$$

Thus, $\left(f_{2}, f_{3}, f_{-2}\right)$ provide a basis for Minkowski space $\mathbb{R}^{2,1}$. Consider the time-like region

$$
V=\left\{x \in \mathbb{R}^{2,1} \mid(x, x)<0\right\},
$$

in $\mathbb{R}^{2,1}$. Let $V^{+}$denote the future light-cone in the space and

$$
\mathbf{Z}=z_{3} f_{2}+z_{2} f_{3}+z_{1} f_{-2}
$$

be such that $\mathbf{Z} \in \mathbb{R}^{2,1}+i V^{+}$. This is equivalent to $\mathbf{Z} \in \mathbb{H}_{2}$, the Siegel upper-half space [4].

Let $M_{1,0}$ be the lattice $\left(\mathbb{Z} f_{2}+\mathbb{Z} f_{3}+\mathbb{Z} f_{-2}\right)$. The root lattice $M_{I I}$ is a sub-lattice in $M_{1,0} . M_{1,0}$ happens to be the root lattice of another rank three hyperbolic KacMoody algebra $[27,28]$. The Weyl group of this algebra is isomorphic to $P G L(2, \mathbb{Z})$. Consider the following identification:

$$
f_{-2} \leftrightarrow\left(\begin{array}{ll}
1 & 0 \\
0 & 0
\end{array}\right), f_{3} \leftrightarrow\left(\begin{array}{ll}
0 & 1 \\
1 & 0
\end{array}\right), f_{2} \leftrightarrow\left(\begin{array}{ll}
0 & 0 \\
0 & 1
\end{array}\right)
$$

The norm of a matrix $N \in M_{1,0}$ is then given by $-2 \operatorname{det} N$. The Weyl group has the following action:

$$
N \rightarrow A \cdot N \cdot A^{T}, \quad A \in P G L(2, \mathbb{Z}) \text { and } N \in M_{1,0} .
$$

Recall that $P G L(2, \mathbb{Z})$ is given by the integral matrices $\left(\begin{array}{ll}a & b \\ c & d\end{array}\right)$ with $a d-b c= \pm 1$. The $S_{3}$ mentioned in Eq. (3.11) is generated by

$$
r_{-1}=\left(\begin{array}{ll}
0 & 1 \\
1 & 0
\end{array}\right) \quad, \quad r_{0}=\left(\begin{array}{cc}
1 & 1 \\
0 & -1
\end{array}\right)
$$

satisfying $r_{-1}^{2}=r_{0}^{2}=\left(r_{-1} r_{0}\right)^{3}=1$. The three elementary reflections that generate $\mathcal{W}\left(A_{1, I I}\right)$ are given by the following $P G L(2, \mathbb{Z})$ matrices:

$$
w_{\delta_{1}}=\left(\begin{array}{cc}
-1 & 0 \\
2 & 1
\end{array}\right), w_{\delta_{2}}=\left(\begin{array}{cc}
1 & 0 \\
0 & -1
\end{array}\right), w_{\delta_{3}}=\left(\begin{array}{cc}
1 & 2 \\
0 & -1
\end{array}\right)
$$




\subsection{The Weyl denominator formula}

\subsubsection{Finite Lie algebras}

The Weyl denominator formula is a specialisation of the Weyl character formula for the trivial representation. For finite Lie algebras, one has 13

$$
\prod_{\alpha \in L_{+}}\left(1-e^{-\alpha}\right)=\sum_{w \in \mathcal{W}} \operatorname{det}(w) e^{[w(\rho)-\rho]}
$$

where $e^{\alpha}$ is a formal exponential and the Weyl vector $\rho$ is defined to be half the sum of all positive roots, i.e., $\rho=\frac{1}{2} \sum_{\alpha \in L_{+}} \alpha$. Further, $w(\rho)$ is the image of $\rho$ under the action of the element $w$ of the Weyl group.

\subsubsection{Affine Kac-Moody algebras}

The first twist occurs for the affine Kac-Moody algebras, where one needs to include imaginary roots, specifically those with zero norm, into the set of positive roots. Thus, one has $L_{+}=L_{+}^{\mathrm{re}} \cup L_{+}^{\mathrm{im}}$ and so on. Further, the imaginary roots appear with multiplicity not necessarily equal to one. For instance, for non-twisted affine algebras, the multiplicity of imaginary roots is equal to the rank of the underlying finite dimensional Lie algebra.

Also, the definition of the Weyl vector as half the sum over all positive roots, needs to be regulated due to the infinite number of such positive roots 14 An alternate definition is given by Lepowsky and Milne which is tailored to writing the sum side of the denominator formula [29]. The key observation (due to MacDonald $[30])$ is that $[w(\rho)-\rho]$ behaves better than either of the terms. Recall that an element of the Weyl group acts as a permutation of all roots (not necessarily positive). Thus, $[w(\rho)-\rho]$ obtains contribution, only when a positive root gets mapped to a non-positive root. So one defines the set $\Phi_{w}$ for all $w \in \mathcal{W}$,

$$
\Phi_{w}=w\left(L_{-}\right) \cap L_{+}=\left\{\alpha \in L_{+} \mid w^{-1}(\alpha) \in L_{-}\right\} .
$$

Using this definition, we can see that

$$
\rho-w(\rho)=\frac{1}{2} \sum_{\alpha \in L_{+}}[\alpha-w(\alpha)] \sim\left\langle\Phi_{w}\right\rangle,
$$

where $\left\langle\Phi_{w}\right\rangle$ is the sum of elements of the set $\Phi_{w}$. Note that $-L_{-}=L_{+}$, which explains the half disappearing in the RHS of the above formula. Imaginary roots

\footnotetext{
${ }^{13}$ Conventionally, the denominator formula is written with $e^{\rho}$ multiplying the form given here. We write in a form that is more suitable to affine Kac-Moody algebras.

${ }^{14}$ There is another definition of the Weyl vector $\rho$, through its inner product with all real simple roots $\delta_{i}$. It is the vector that satisfies $\left(\rho, \delta_{i}\right)=\frac{1}{2}\left(\delta_{i}, \delta_{i}\right), \forall i$.
} 
do not appear in the set $\Phi_{w}$ for affine Lie algebras as the imaginary roots turn out to be Weyl invariant and hence cancel out in the above equation.

The denominator formula that works for affine Kac-Moody algebras, after including the imaginary roots in $L_{+}$, is the Weyl-Kac denominator formula

$$
\prod_{\alpha \in L_{+}}\left(1-e^{-\alpha}\right)^{\operatorname{mult}(\alpha)}=\sum_{w \in \mathcal{W}} \operatorname{det}(w) e^{-\left\langle\Phi_{w}\right\rangle}
$$

For the affine Kac-Moody algebra, $\widehat{A}_{1}^{(1)}$, from the above definition of the set of positive roots, we have

$$
L_{+}=\left(n\left(\alpha_{1}+\alpha_{0}\right), n \alpha_{1}+(n-1) \alpha_{0},(n-1) \alpha_{1}+n \alpha_{0} \mid n=1,2,3, \ldots\right),
$$

and the Weyl group is isomorphic to $\mathbb{Z}_{2} \ltimes \mathbb{Z}$. Putting it all together into the denominator identity gives [29]

$$
\begin{aligned}
& \prod_{n \geq 1}\left(1-e^{-n \alpha_{0}} e^{-n \alpha_{1}}\right)\left(1-e^{-(n-1) \alpha_{0}} e^{-n \alpha_{1}}\right)\left(1-e^{-n \alpha_{0}} e^{-(n-1) \alpha_{1}}\right) \\
& =\sum_{n \in \mathbb{Z}} e^{-n(2 n-1) \alpha_{0}} e^{-n(2 n+1) \alpha_{1}}-\sum_{n \in \mathbb{Z}} e^{-(n+1)(2 n+1) \alpha_{0}} e^{-n(2 n+1) \alpha_{1}}
\end{aligned}
$$

Setting $e^{-\alpha_{0}}=r$ and $e^{-\alpha_{1}}=q r^{-1}$, the above identity is equivalent to the Jacobi triple identity involving the theta function $\vartheta_{1}(\tau, z)$ :

$$
\begin{aligned}
-i \vartheta_{1}(\tau, z) & =q^{1 / 8} r^{-1 / 2} \prod_{n=1}^{\infty}\left(1-q^{n}\right)\left(1-q^{n-1} r\right)\left(1-q^{n} r^{-1}\right) \\
& =\sum_{n \in \mathbb{Z}}(-1)^{n} q^{\frac{(n-1 / 2)^{2}}{2}} r^{n-1 / 2} .
\end{aligned}
$$

\subsubsection{Generalized Kac-Moody algebras}

For the case of generalized Kac-Moody algebras, the story gets a bit more involved. A detailed discussion of the Weyl denominator formula for GKM algebras can be found in [31]. New kinds of roots appear here and have nontrivial multiplicities that are hard to determine. The 'sum side' denominator formula also requires modification. For the class of GKM algebras with a bilinear form that is almost positive definite the denominator identity was constructed by Borcherds generalizing the Weyl-Kac character formula for Kac-Moody algebras [11,32-34]. These GKM algebras contain, in addition to real and imaginary roots of Kac-Moody algebras, imaginary simple roots and a suitable definition of the denominator identity is required to correct for these. Also, the way the Cartan subalgebra is centrally 
extended modifies the root space and requires some care as the linear independence of the simple roots is crucial for the denominator identity to be well defined [34]. The Weyl group continues to be generated only by elementary reflections of the real simple roots.

For the above class of generalized Kac-Moody algebras, the Weyl-Kac-Borcherds denominator identity becomes [32]

$$
\prod_{\alpha \in L_{+}}\left(1-e^{-\alpha}\right)^{\operatorname{mult}(\alpha)}=e^{-\rho} \sum_{w \in \mathcal{W}}(\operatorname{det} w) w\left(e^{\rho} \sum_{\alpha \in L_{+}} \epsilon(\alpha) e^{\alpha}\right)
$$

where $L_{+}$is the set of positive roots, $\rho$ the Weyl vector, $\mathcal{W}$ the Weyl group of the GKM, $\operatorname{det}(w)$ is defined to be \pm 1 depending on whether $w$ is the product of an even or odd number of reflections and $\epsilon(\alpha)$ is defined to be $(-1)^{n}$ if $\alpha$ is the sum of $n$ pairwise independent, orthogonal imaginary simple roots, and 0 otherwise. As we will consider superalgebras, the above formula, Eq. (3.29) continues to hold with roots appearing with graded multiplicity - fermionic roots appear with negative multiplicity.

As before we can cast it into a slightly different form by constructing the set

$$
\Phi_{w}=w\left(L_{-}\right) \cap L_{+}=\left\{\alpha \in L_{+} \mid w^{-1}(\alpha) \in L_{-}\right\} .
$$

such that

$$
\rho-w(\rho)=\left\langle\Phi_{w}\right\rangle
$$

and the denominator identity takes the form [34]

$$
\prod_{\alpha \in L_{+}}\left(1-e^{-\alpha}\right)^{\operatorname{mult}(\alpha)}=\sum_{w \in W^{\sigma}}(\operatorname{det} w) \sum_{\eta \in \Omega}(-1)^{\mathrm{ht}(\eta)} e^{-\left\langle\Phi_{w}\right\rangle-w(\eta)}
$$

where $\Omega$ is defined as the sum of all the possible sets of distinct pairwise orthogonal imaginary simple roots and the height ht $(\eta)$ of an element $\eta=\sum_{i} n_{i} \delta_{i}$ is $\sum_{i} n_{i}$. We will verify that the modular forms that we construct have product and sum representations that are indeed compatible with the denominator formula.

\section{Denominator formulae for $\Delta_{k / 2}(\mathbf{Z})$}

Gritsenko and Nikulin have shown that the denominator formula of a GKM superalgebra $\mathcal{G}_{1}$ is related to a modular form of $\operatorname{Sp}(2, \mathbb{Z})$ with character, $\Delta_{5}(\mathbf{Z})$. The modular form, $\Phi_{10}(\mathbf{Z})$, that generates the degeneracy of dyons is equal to $\Delta_{5}(\mathbf{Z})^{2}$. We extend this correspondence to argue for the existence of new GKM superalgebras, $\mathcal{G}_{N}$, whose denominator formulae give rise to modular forms with character, $\Delta_{k / 2}(\mathbf{Z})$, of suitable groups, $G_{0}(N) \subset \operatorname{Sp}(2, \mathbb{Z})$, with the property

$$
\Delta_{k / 2}(\mathbf{Z})^{2}=\Phi_{k}(\mathbf{Z}) \text {. }
$$


(Note that the $\Phi_{k}(\mathbf{Z})$ used here differs from the one defined by David-JatkarSen [17] by an overall sign.) This is the main assumption of this paper. Recall that the modular forms $\Phi_{k}(\mathbf{Z})$ were first constructed in [9] and product formulae were provided in subsequent papers $[17,22]$. Further, the dyon degeneracies are given by a closely related modular form called $\tilde{\Phi}_{k}(\mathbf{Z})$ by Jatkar and Sen. This differs in the way the S-duality group $\Gamma_{1}(N) \subset S L(2, \mathbb{Z})$ is embedded in $S p(2, \mathbb{Z})$.

\subsection{Constructing the modular form $\Delta_{k / 2}(\mathrm{Z})$}

We have found experimentally that the modular form $\Delta_{k / 2}(\mathbf{Z})$ can be obtained as the additive lift of the Jacobi cusp form of $\Gamma_{1}(N)$ of weight $k / 2$ and index $1 / 2$

$$
\psi_{k / 2,1 / 2}(\tau, z)=\theta_{1}(\tau, z) \eta(\tau)^{(k-4) / 2} \eta(N \tau)^{(k+2) / 2} .
$$

A proof of the additive lift has been given in appendix C.

Note that in the following we will write $z_{1}$ for $\tau$ and $z_{2}$ for $z$ keeping in mind that these become two of the three coordinates on $\mathbb{H}_{2}$. This happens to be the square root of the Jacobi form that generates $\Phi_{k}(\mathbf{Z})$. A discussion of the additive lift has been provided in appendix B. The Fourier expansion

$$
\psi_{k / 2,1 / 2}\left(z_{1}, z_{2}\right)=\sum_{n, \ell \equiv 1 \bmod 2} g(n, \ell) q^{n / 2} r^{\ell / 2},
$$

with $q=\exp \left(2 \pi i z_{1}\right)=\exp (2 \pi i \tau)$ and $r=\exp \left(2 \pi i z_{2}\right)=\exp (2 \pi i z)$, enables us to define the additive lift for $\Delta_{k / 2}(\mathbf{Z})$ :

$$
\Delta_{k / 2}(\mathbf{Z}) \equiv \sum_{(n, \ell, m)>0} \sum_{d \mid(n, \ell, m)} \chi(d) d^{\frac{k-2}{2}} g\left(\frac{n m}{d^{2}}, \frac{\ell}{d}\right) q^{n / 2} r^{\ell / 2} s^{m / 2},
$$

where $s=\exp \left(2 \pi i z_{3}\right)$. This generalises the Maaß formula for $\Delta_{5}(\mathbf{Z})$ when $(N, k)=$ $(1,10)$. We have experimentally verified that the square of the above formula generates $\Phi_{k}(\mathbf{Z})$ to fairly high order (all terms which appear with values of $m n \leq 15$ in the Fourier expansion for $\Phi_{k}(\mathbf{Z})$ and more to confirm the non-trivial character for $N=3$ ). We find that for $N=2,5$, the character $\chi(d)$ is the trivial one (see Eq. (B.16) $)$ while for $N=3$, we need a non-trivial character $\chi^{\psi}(d)=\left(\frac{-3}{d}\right)$ i.e.,

$$
\chi^{\psi}(d)=\left\{\begin{array}{rll}
0 & d=0 & \bmod 3, \\
1 & d=1 & \bmod 3, \\
-1 & d=2 & \bmod 3 .
\end{array}\right.
$$

It is known that $\Delta_{5}(\mathbf{Z})$ can be written as a product of all ten even genus-two theta constants. As we show in the appendix, $\Delta_{3}(\mathbf{Z})$ can be written as a product of six even genus-two theta constants:

$$
\Delta_{3}(\mathbf{Z})=\frac{1}{64} \theta_{2}(\mathbf{Z}) \prod_{m=1 \bmod 2} \theta_{m}(\mathbf{Z})
$$


We however do not expect such a formula for the other $\Delta_{k / 2}(\mathbf{Z})$.

It is of interest to ask whether these modular forms already exist in the mathematical literature. For $N=2$, we have been able to show that $\Delta_{3}(\mathbf{Z})$ is given by the product of six even genus-two theta constants. In fact, the square root of $\tilde{\Phi}_{6}(\mathbf{Z})$, which we call $\tilde{\Delta}_{3}(\mathbf{Z})$, can also be written as the product of six (different) even genus-two theta constants (see appendix A.2 and ref. [35,36]). Aoki and Ibukiyama [35] have studied the ring of modular forms at levels $N=2,3,4$. At level $N=3$, they show that modular forms with character,

$$
v^{\psi}(M)=\left(\frac{-3}{\operatorname{det}(D)}\right)
$$

for $M=\left(\begin{array}{cc}A & B \\ C & D\end{array}\right) \in \widehat{G}_{0}(3)$, necessarily have odd weight. However, for $N=3$, we obtain a modular form with even weight, $\Delta_{2}(\mathbf{Z})$. Is there a contradiction? The resolution lies in the fact that $\Delta_{2}(\mathbf{Z})$ transforms with a different character 15 - it is the one given by the product of the character, $v^{\psi}(M)$, considered by Aoki-Ibukiyama and the restriction of the character, $v^{\Gamma}(M)$, that appears in the transformation of $\Delta_{5}(\mathbf{Z})$, to $\widehat{G}_{0}(3)$ (see appendix B.4). In appendix A.1, we show that the Jacobi form that generates $\Delta_{2}(\mathbf{Z})$ is one with non-trivial character which is consistent with our expectations. This combined with the additive lift shown in appendix $C$ show that this indeed true.

With the construction of $\Delta_{k / 2}(\mathbf{Z})$, we are ready to verify whether they can be the denominator formula of a GKM superalgebra $\mathcal{G}_{N}$. The product formula for $\Delta_{k / 2}(\mathbf{Z})$ can be obtained from Eq. (2.13) and it gives us the positive roots $L_{+}$ with their multiplicities.

$\Delta_{k / 2}(\mathbf{Z})=q^{1 / 2} r^{1 / 2} s^{1 / 2} \prod_{n, \ell, m \in \mathbb{Z}}\left(1-q^{n} r^{\ell} s^{m}\right)^{\frac{1}{2} c_{1}(n m, \ell)} \times \prod_{n, \ell, m \in \mathbb{Z}}\left(1-\left(q^{n} r^{\ell} s^{m}\right)^{N}\right)^{\frac{1}{2} c_{2}(n m, \ell)}$.

For this to make sense as the product side of a denominator formula of a GKM superalgebra, it is necessary that $c_{1}(n m, \ell)$ and $c_{2}(n m, \ell)$ are even integers as they provide the multplicity of various roots in $L_{+}$. It appears to be true to the orders that we have checked. The common factor $q^{1 / 2} r^{1 / 2} s^{1 / 2}$ can be identified with $\exp (-\pi \imath(\rho, z))$ giving us the Weyl vector $\rho$. We thus see that the Weyl vector is independent of $N$. The $\rho$ vector does not change during the orbifolding process in the work of Niemann either (see Theorem (1.6) in ref. [12]). In the work of

\footnotetext{
${ }^{15}$ We thank Prof. Aoki for encouraging us to believe in the existence of $\Delta_{2}(\mathbf{Z})$ even though our original approach initially had no mathematical rigor. He has also informed us that Gritsenko has independently constructed a modular form of weight two at level 3. We also thank D. Ramakrishnan for a useful conversation.
} 
Niemann, the orbifolding changes the simple roots whereas in our situation, the three real simple roots are unaffected by the orbifolding.

However, one needs to verify that the Maaß formula (4.4) corresponds to the sum side of the denominator formula. This is the procedure adopted by Gritsenko and Nikulin for $\Delta_{5}(\mathbf{Z})$ and we repeat their method [4].

\subsection{The GKM superalgebra $\mathcal{G}_{1}$}

We first consider the example of $\mathcal{G}_{1}$ to illustrate the method before moving on to the new GKM superalgebras. As it stands, the denominator formula for the Lie algebra $\mathfrak{g}\left(A_{1, I I}\right)$ is not related to the the automorphic form $\Delta_{5}(\mathbf{Z})$. Following the ideas of Borcherds [11,32], Gritsenko and Nikulin constructed a superalgebra, $\mathcal{G}_{1}$ by adding imaginary simple roots - some are bosonic and others are fermionic. The superalgebra $\mathcal{G}_{1}$ has $\mathfrak{g}\left(A_{1, I I}\right)$ as a sub-algebra and its Weyl group is identical to the one for $\mathfrak{g}\left(A_{1, I I}\right)$.

In the above setting, the Weyl-Kac-Borcherds denominator formula 16 (3.29) becomes (see also sec. 3.3.1)

$$
\begin{aligned}
e^{-\pi \imath(\rho, z)} & \prod_{\alpha \in L_{+}}\left(1-e^{-\pi \imath(\alpha, z)}\right)^{\operatorname{mult}(\alpha)} \\
& =\left(\sum_{w \in \mathcal{W}} \operatorname{det}(w)\left\{e^{-\pi \imath(w(\rho), z)}-\sum_{\eta \in M_{I I} \cap \mathbb{R}_{+} \mathcal{M}_{I I}} m(\eta) e^{-\pi \imath(w(\rho+\eta), z)}\right\}\right)
\end{aligned}
$$

where the element $\mathbf{Z}=z_{3} f_{2}+z_{2} f_{3}+z_{1} f_{-2}$ belongs to the subspace $\mathbb{R}^{2,1}+\imath V^{+} \sim \mathbb{H}_{2}$ obtained upon complexification of the cone $V^{+}$. We have deliberately rewritten the sum as two terms - one arising from the real simple roots $(\eta=0)$ and the other arising from the imaginary simple roots $(\eta \neq 0)$. The first term thus arises as the sum side of the Lie algebra $\mathfrak{g}\left(A_{1, I I}\right)$. The second terms is specific to GKM algebras due to the presence of imaginary simple roots with 'multiplicities' $m(\eta) \in \mathbb{Z}$. These multiplicities are determined by the connection with the automorphic form $\Delta_{5}(\mathbf{Z})$ (an explicit expansion is given in appendix D) - in other words, one adds enough imaginary simple roots such that the automorphic properties are attained. The imaginary simple roots belong to the space $M_{I I} \cap \mathbb{R}_{+} \mathcal{M}_{I I}$. The Maaß formula for $\Delta_{5}(\mathbf{Z})$ leads to a simple expression for light-like imaginary simple roots i.e., $\left(\eta_{0}, \eta_{0}\right)=0$. They are generated by the formula

$$
1-\sum_{t \in \mathbb{N}} m\left(t \eta_{0}\right) q^{t}=\prod_{k \in \mathbb{N}}\left(1-q^{k}\right)^{9}=\frac{\sqrt{f^{(10)}(\tau)}}{\eta(\tau)^{3}} .
$$

\footnotetext{
${ }^{16}$ Here we write the denominator formula in the notation of Gritsenko and Nikulin. In particular, one needs to replace $\rho$ by $-\rho$ in Eq. (3.29).
} 
Negative values of multiplicity implies that the root is fermionic. For instance, one has $m\left(2 \eta_{0}\right)=-27$. Thus, such roots are fermionic and hence we have a superalgebra. There are three primitive light-like vectors: $2 f_{2}, 2 f_{-2}$ and $\left(2 f_{-2}-\right.$ $2 f_{3}+2 f_{2}$ ) each with multiplicity 9. The action of the Weyl group generates the remaining vectors. There are two primitive vectors satisfying $(\eta, \eta)<0$ : $\left(2 f_{-2}+2 f_{2}\right)$ and $\left(2 f_{-2}-f_{3}+2 f_{2}\right)$. The other terms are generated as multiples of the form $t \eta$ of these primitive vectors and multiplicities given by the above formula.

The product formula for $\Delta_{5}(\mathbf{Z})$ determines the positive roots $L_{+}-$again fermionic roots appear with negative multiplicity in the exponent. We do not write out the detailed list and refer the diligent reader to the paper by Gritsenko and Nikulin [4]. We would like to comment here that there is a subtle issue in extracting the multiplicities from the exponent in the product formula - the product formula gives only the difference between the multiplicities of the bosonic and fermionic generators and hence is more like a Witten index.

\subsection{The GKM superalgebra $\mathcal{G}_{2}$}

An important assumption in this paper has been that the square root of the Siegel modular forms $\Phi_{k}(\mathbf{Z})$, i.e., $\Delta_{k / 2}(\mathbf{Z})$ should appear as the denominator formula of some GKM superalgebra. The first example that we use to verify this is the $\mathbb{Z}_{2}$ orbifold for which $k=6$. It might be thought that although $\Phi_{10}(\mathbf{Z})$ and $\Phi_{6}(\mathbf{Z})$ are not obviously associated to any GKM algebras, we could nevertheless obtain the changes that result from the orbifolding by directly comparing the two as given by their Maaß expansions. The remarkable thing is that the comparison at the level of the modular forms, $\Phi_{10}(\mathbf{Z})$, and $\Phi_{6}(\mathbf{Z})$, is not very transparent, in that the terms appearing in the two are not one-to-one for comparison. There occur terms in each that do not appear in the other, and hence we can not track down their fate as we orbifold. It is only when we compare $\Delta_{5}(\mathbf{Z})$ and $\Delta_{3}(\mathbf{Z})$ we see that both the expansions are similar and suitable for comparison. Below we analyze the Maaß expansion of its square root, $\Delta_{3}(\mathbf{Z})$, in the same way as was done for $\Delta_{5}(\mathbf{Z})$ and obtain the multiplicities of the primitive imaginary simple roots $\eta$.

We have already derived a Maaß formula for $\Delta_{3}(\mathbf{Z})$ using the additive lift of the weak Jacobi form $\psi_{3,1 / 2}\left(z_{1}, z_{2}\right)$ of weight 3 and index $1 / 2$ as discussed earlier. We also need to suitably interpret the region of summation of the variables $n, \ell, m$ such that they give the space $\Omega$ of the algebra we construct. Mathematically, these will be the 'twisted' generalized GKM algebras that are obtained by the orbifold action on $\mathcal{G}_{1}$ analogous to the ones constructed by Niemann [12].

A straightforward comparison of the Fourier expansions $\Delta_{3}(\mathbf{Z})$ and $\Delta_{5}(\mathbf{Z})$ (given in Appendix D) by first focusing on terms appearing from real simple roots, we observe that the Cartan matrix for $\mathcal{G}_{2}$ is the same as for $\mathcal{G}_{1}$. Next, we can see 
that all terms that appear in $\Delta_{5}(\mathbf{Z})$ appear in $\Delta_{3}(\mathbf{Z})$ albeit with different coefficients. Thus the set of simple roots - real and imaginary, of $\mathcal{G}_{2}$ remain unchanged from $\mathcal{G}_{1}$. This implies the lattice generated by the three real simple roots $M_{I I}=$ $\mathbb{Z} \delta_{1} \oplus \mathbb{Z} \delta_{2} \oplus \mathbb{Z} \delta_{3}$ is the same as before. It also implies the cone defined by the lattice $V^{+}\left(M_{I I}\right)$ and the fundamental polyhedron $\mathcal{M}_{I I}$, generated by the spaces bounded by the real roots, also remain unchanged. The fundamental cone, $M_{I I} \cap \mathbb{R}_{+} \mathcal{M}_{I I}$, containing the the imaginary roots also remains unchanged by orbifolding. As already observed, the Weyl vector for the lattice $\rho$ is the same as for $\mathcal{G}_{1}$.

The only difference comes in the generating function of the multiplicity factors such as $m\left(\eta_{0}\right)$. The $m\left(\eta_{0}\right)$ in the case of $\mathcal{G}_{2}$ are given by the generating function

$$
1-\sum_{t \in \mathbb{N}} m\left(t \eta_{0}\right) q^{t}=\prod_{k \in \mathbb{N}}\left(1-q^{k}\right)\left(1-q^{2 k}\right)^{4}=\frac{\sqrt{f^{(6)}(\tau)}}{\eta(\tau)^{3}}
$$

This can be understood from the twisted denominator formula of Niemann [12] for the cycle shape $1^{k+2} N^{k+2}$ for $N=2$, and $k=6$ and is the special case of a more general formula for any $N$ and $k$ which we give below.

It is remarkable that although the degeneracy of the dyons are given by completely different modular forms in the two cases, the underlying GKM that we can construct for them are similar to such a degree. The roots - real and imaginary, Weyl group, Weyl vector, the lattice, and the space of the imaginary roots remain unchanged. The orbifolding of the space is reflected only in the change of the multiplicity of the imaginary roots.

\subsection{The GKM superalgebras $\mathcal{G}_{N}$}

In this section we list the properties of the class of GKM algebras $\mathcal{G}_{N}$ for $N=$ $1,2,3,5$ obtained from the modular forms that occur in degeneracy formulae for a $\mathbb{Z}_{N}$ orbifolding action.

The Cartan matrix, Weyl group, and the set of real and imaginary simple roots for the $\mathcal{G}_{N}$ remain the same for all values of $N$. The modular forms leading to these algebras, and hence the denominator identities of the algebras, however, are different from each other. The difference in the denominator identities is in the coefficients of the terms occurring in the expansion, whereas the terms themselves undergo no change.

The generating functions of the multiplicity factors of light-like simple roots for different values of $N$ are given in terms of a single formula 17 :

$$
\theta_{1}(\tau, z)\left(1-\sum_{t \in \mathbb{N}} m\left(t \eta_{0}\right) q^{t}\right)=\psi_{k / 2,1 / 2}(\tau, z) .
$$

\footnotetext{
${ }^{17}$ Interestingly, it was this pattern that lead us to the proposed additive lift using the weak Jacobi form $\psi_{k / 2,1 / 2}\left(z_{1}, z_{2}\right)$.
} 
From the above we see the pattern in the progression of the $m\left(\eta_{0}\right)$ as the orbifolding group $\mathbb{Z}_{N}$ varies. For $N=3$, one sees that certain multiplicities vanish and thus there are fewer terms in the Fourier expansion for $\Delta_{3}(\mathbf{Z})$ as can be seen in appendix D.

The additive lift for $N=5$ is generated by a weak Jacobi form. While the formula appears to go through, there are issues with the convergence of the series. This affects the holomorphicity of both the forms $-\Phi_{2}(\mathbf{Z})$ and $\Delta_{1}(\mathbf{Z})$. However, these forms (and their modular transforms) appear to be compatible with the entropy of black holes as well as the higher-derivative $R^{2}$-corrections in the lowenergy effective action. It is not clear to us whether this meromorphicity of $\Delta_{1}(\mathbf{Z})$ affects the existence of the GKM superalgebra $\mathcal{G}_{5}$ but we caution the reader about this possibility.

One may wonder what goes wrong when $N=7$. The weak Jacobi form has half-integral weight as well as half-integral index. It does not have an integral Fourier expansion and hence does not seem to lead to a GKM superalgebra with integer multiplicities. There is a related issue - the modular form $\Phi_{1}(\mathbf{Z})$ is one with character and hence we anticipates subtleties associated with it.

\subsection{Interpreting the Jacobi form $\psi_{k / 2,1 / 2}\left(z_{1}, z_{2}\right)$}

Given a Siegel modular form, $F_{k}(\mathbf{Z})$ of weight $k$ with $\mathbf{Z} \in \mathbb{H}_{2}$, its Fourier expansion with respect to $z_{3}$ can be written as

$$
F_{k}(\mathbf{Z})=\sum_{m=0}^{\infty} \phi_{k, m}\left(z_{1}, z_{2}\right) \exp \left(2 \pi i m z_{3}\right)
$$

For cusp forms, the term $m=0$ vanishes. The Fourier coefficients $\phi_{k, m}\left(z_{1}, z_{2}\right)$ are weak Jacobi forms of weight $k$ and index $m$ under the sub-group, $\Gamma^{J}$ of $S p(2, \mathbb{Z})$ under which the cusp $\operatorname{Im}\left(z_{3}\right)=\infty$ is invariant(see appendix $\mathbb{B}$ ). For Siegel modular forms with character such as $\Delta_{5}(\mathbf{Z})$, the above sum runs over half-integers and we obtain Jacobi forms with half-integral index.

In our situation, taking the $\operatorname{limit} \operatorname{Im}\left(z_{3}\right) \rightarrow \infty$ can also be understood as the removal of a real root of the GKM superalgebra $\mathcal{G}_{N}$. The Cartan matrix for the two-dimensional subspace of real roots is then the same as the affine Kac-Moody algebra $\hat{A}_{1}^{(1)}$ at level one. We thus see that $\hat{A}_{1}^{(1)} \subset \mathcal{G}_{N}$. As is well known and discussed earlier, the denominator formula for this algebra is given by $i \theta_{1}\left(z_{1}, z_{2}\right)$. In the product form for the theta function, it is easy to see the appearance of imaginary roots with multiplicity one. The Jacobi forms of $\Gamma_{0}(N)$ that we are interested in contain other powers of $\eta$ - these are reflected in the fact that the $\mathcal{G}_{N}$ has more light-like imaginary roots than $\hat{A}_{1}^{(1)}$ - it is these roots that appear in the correction terms in the sum side of the denominator formula. For instance, the 
imaginary simple root $\eta_{0}=2 f_{-2}$ has multiplicity $m\left(\eta_{0}\right)=9$ for $N=1$. The same root appears in $L_{+}$with multiplicity 10 . The difference is easy to understand the affine Lie algebra has one light-like root that is not simple - it is the sum of the two real roots. For $N=3,5$, we find $m\left(\eta_{0}\right)=0,-1$ respectively. So we see that the root $\eta_{0}$ is a fermionic one for $N=5$.

As mentioned earlier, the analysis of David and Sen using the 4D-5D lift leads to a separation of the product formula for $\Phi_{k}(\mathbf{Z})$ into three terms. Taking the square-root to obtain $\Delta_{k / 2}(\mathbf{Z})$ does not change the separation. We see that two of the terms are nothing but the product representation for the weak Jacobi form of index $\frac{1}{2}, \psi_{k / 2,1 / 2}\left(z_{1}, z_{2}\right)$. It appears that real and light-like simple roots appear from the spacetime and $T^{2}$ sectors in the type II picture. In particular, the electrically charged heterotic string states appear to arise from such light-like simple roots. We believe that this is a small step in understanding the connection between the GKM superalgebras and the algebra of BPS states.

\section{Conclusion and Outlook}

In this paper, we have shown that the square-root of the automorphic form $\Phi_{k}(\mathbf{Z})$ that generates the degeneracy of 1/4-BPS CHL dyons can be interpreted as the Weyl-Kac-Borcherds denominator formula for a GKM superalgebra. Further, we have proposed an additive lift that directly generates the automorphic form $\Delta_{k}(\mathbf{Z})$ from a weak Jacobi form of index $1 / 2$. From the physical point of view, using the 4D-5D lift, we have been able to show that the real roots and light-like imaginary roots for electrically charged states arise from spacetime and $T^{2}$ sectors (in the type II picture) while the other imaginary roots necessarily arise from the $K 3$ sector.

Cheng and Verlinde [7] observe that the walls of the Weyl chambers for the GKM superalgebra $\mathcal{G}_{1}$ get mapped to walls of marginal stability for the 1/4-BPS dyons [37-40]. This observation, if extended to the $\mathbb{Z}_{N}$-orbifolds, seems, at first sight, to be in contradiction with our observation that the Weyl group remained unchanged for $\mathcal{G}_{N}$. However this naive extension is not quite correct, since for $N>1$, there are two distinct modular forms that have been constructed by JatkarSen [9], $\Phi_{k}(\mathbf{Z})$ and $\widetilde{\Phi}_{k}(\mathbf{Z})$. The first one is related to the $R^{2}$-corrections in the low-energy effective action while the second one is the one that generates the degeneracy of 1/4-BPS dyons. Walls of marginal stability are precisely where this degeneracy jumps. The extension of the Cheng and Verlinde observation should be applicable to GKM superalgebras related to the square-root of $\widetilde{\Phi}_{k}(\mathbf{Z})$. This analysis has been recently carried out by Cheng and Dabholkar [41] who find that the Weyl groups of the correspoding GKM superalgebras change with $N$ and the walls of the Weyl chambers get mapped to the walls of marginal stability for 
$N=2,3$.

Sen has studied the fundamental domain in the upper half-plane bounded by walls of marginal stability [37] and from his results one sees that for $N>3$, the domain has infinite volume (in the Poincaré metric). Cheng and Dabholkar also observe that the $N=5$ situation violates a certain finiteness condition (imposed by Gritsenko and Nikulin in their classification of rank three Lorentzian Kac-Moody algebras [42]) and thus they conclude that there is no GKM superalgbra for $N=5$. Should one relax the finiteness condition and look for a GKM superalgebra for $N=5$ ? Is the meromorphicity of the modular form that we observed for $N=5$ relevant? We do not have any concrete answers to these questions and we leave if for future considerations.

Garbagnati and Sarti have studied symplectic (Nikulin) involutions of K3 manifolds [43,44]. In particular, they have explicitly constructed elliptic K3's whose automorphism groups are the Nikulin involutions. Further, they have provided an explicit description of the invariant lattice and its complementary lattice. We anticipate that these results might be relevant in improving our physical understanding the role of the roots of the GKM superalgebras. The Jatkar-Sen construction holds for $N=11$ as well and it leads to a modular function (i.e., one of weight $k=0$ ) $\Phi_{0}(\mathbf{Z})$ and it is believed that a CHL string may exist. In the type IIA picture, the $\mathbb{Z}_{11}$ is no longer a symplectic Nikulin involution, it acts non-trivially on $H^{*}(K 3)$ and not on $H^{1,1}(K 3)$ alone. It is of interest to study aspects of the $\mathbb{Z}_{N}$ orbifold both from the physical and mathematical point of view.

As mentioned in the introduction, our aim has been to address the algebra of BPS states. While we have uncovered a nice algebraic structure, no direct relationship to the algebra of BPS states has been achieved. A related problem is that the degeneracy of BPS states appears related to the direct sum of two copies of the GKM superalgebras, $\mathcal{G}_{N}$. BPS states thus seem to be elements of a module that is a tensor product of two copies of (irreducible?) representations of the superalgebra. It would be nice to have a microscopic understanding of these issues. A possibly relevant observation here is that the elliptic genus of the Enriques surface [45], these are two-dimensional complex surfaces that arise as fixed-point free $\mathbb{Z}_{2}$ involutions of K3 surfaces, gives rise to $\Delta_{5}(\mathbf{Z})$. This might provide a hint on the appearance of two identical copies of the same GKM superalgebra for K3.

As we have seen, for affine Kac-Moody algebras, the presence of light-like imaginary roots in $L_{+}$leads to powers of the Dedekind eta function appearing in the product form of the Weyl-Kac denominator formula. As is well known, $q^{1 / 24} / \eta(\tau)$ is the generating function of partitions of $n$ (equivalently, Young diagrams with $n$ boxes). An interesting generalisation is the generating function of plane partitions (or 3D Young diagrams) has a nice product representation $\eta_{3 D} \sim \prod_{n}\left(1-q^{n}\right)^{n}$ (due to MacMahon). This function appears in the counting of D0-branes in the 
work of Gopakumar-Vafa [46, 47]. Is there an algebraic interpretation for this? The addition of D2-branes to this enriches this story and leads to interesting formulae [48].

Acknowledgments: We would like to thank Dileep Jatkar and Hiroki Aoki for patiently answering our numerous queries via email. KGK would like to thank S. Kalyana Rama for his support. SG would like to thank the CERN Theory Group for a visit during which some of this work was carried out. We thank the anonymous referee for critical comments that have led to several improvements in our paper.

\section{A Theta functions}

\section{A.1 Genus-one theta functions}

The genus-one theta functions are defined by

$$
\theta\left[\begin{array}{l}
a \\
b
\end{array}\right]\left(z_{1}, z_{2}\right)=\sum_{l \in \mathbb{Z}} q^{\frac{1}{2}\left(l+\frac{a}{2}\right)^{2}} r^{\left(l+\frac{a}{2}\right)} e^{i \pi l b}
$$

where $a . b \in(0,1) \bmod 2$. One has $\vartheta_{1}\left(z_{1}, z_{2}\right) \equiv \theta\left[\begin{array}{l}1 \\ 1\end{array}\right]\left(z_{1}, z_{2}\right), \vartheta_{2}\left(z_{1}, z_{2}\right) \equiv \theta\left[\begin{array}{l}1 \\ 0\end{array}\right]\left(z_{1}, z_{2}\right)$, $\vartheta_{3}\left(z_{1}, z_{2}\right) \equiv \theta\left[\begin{array}{l}0 \\ 0\end{array}\right]\left(z_{1}, z_{2}\right)$ and $\vartheta_{4}\left(z_{1}, z_{2}\right) \equiv \theta\left[\begin{array}{l}0 \\ 1\end{array}\right]\left(z_{1}, z_{2}\right)$.

The transformations of $\vartheta_{1}(\tau, z)$ under modular transformations is given by

$$
\begin{aligned}
T: & \vartheta_{1}(\tau+1, z)=e^{i \pi / 4} \vartheta_{1}(\tau, z), \\
S: & \vartheta_{1}(-1 / \tau,-z / \tau)=-\frac{1}{q^{1 / 2} r} e^{\pi i z^{2} / \tau} \vartheta_{1}(\tau, z),
\end{aligned}
$$

with $q=\exp (2 \pi i \tau)$ and $r=\exp (2 \pi i z)$.

The Dedekind eta function $\eta(\tau)$ is defined by

$$
\eta(\tau)=e^{2 \pi i \tau / 24} \prod_{n=1}^{\infty}\left(1-q^{n}\right) .
$$

The transformation of the Dedekind eta function under the modular group is given by

$$
\begin{array}{ll}
T: & \eta(\tau+1)=e^{\pi i / 12} \eta(\tau), \\
S: & \eta(-1 / \tau)=e^{-\pi i / 4}(\tau)^{1 / 2} \eta(\tau) .
\end{array}
$$


The transformation of $\eta(N \tau)$ is given by

$$
\begin{aligned}
T: & \eta(N \tau+N) & =e^{N \pi i / 12} \eta(\tau) \\
S: & \eta(-1 / \tau) & =\frac{e^{-\pi i / 4}}{\sqrt{N}}(\tau)^{1 / 2} \eta(\tau / N) .
\end{aligned}
$$

One can see that $\eta(N \tau)$ transforms into $\eta(\tau / N)$ under the $S$ transformation. $\eta(N \tau)$ gets mapped to itself only under the subgroup, $\Gamma_{0}(N)$ of $S L(2, \mathbb{Z})$. Following Niemann [12], let

$$
\psi_{j}(\tau) \equiv \eta\left(\frac{\tau+j}{N}+j\right), j=0,1, \ldots, N-1 \quad \bmod N .
$$

Both $S$ and $T$ no longer have a diagonal action on the $\psi_{j}(\tau)$. One has

$$
\begin{array}{ll}
T: & \psi_{j}(\tau+1)=e^{\pi i / 12} \psi_{j+1}(\tau) \\
S: & \psi_{j}(-1 / \tau)=e^{\left(j+j^{\prime}\right) \pi i / 12}(\tau)^{1 / 2} \chi(G) \psi_{-j^{\prime}}(\tau),
\end{array}
$$

where $j j^{\prime}=1 \bmod N$ and the character $\chi(G)$ has to be calculated on a case by case basis (see chapter 2 of [12] for details).

The transformations of the eta related functions show us that the functions $f^{k}(\tau)$ and its square root can transform with non-trivial character. In particular, one can show that for $N=7, f^{(1)}(\tau)$ and for $N=3, \sqrt{f^{(4)}(\tau)}$ transform with character. As these two functions enter the weak Jacobi forms that are used to construct the Siegel modular forms $\Phi_{1}(\mathbf{Z})$ and $\Delta_{2}(\mathbf{Z})$ respectively, these two Siegel modular forms will transform with non-trivial character [50]. This is the basis for our claim that $\Delta_{2}(\mathbf{Z})$ must transform with non-trivial character and is consistent with the observation of Jatkar-Sen regarding $\Phi_{1}(\mathbf{Z})$.

\section{A.2 Genus-two theta constants}

We define the genus-two theta constants as follows [4]:

$$
\theta\left[\begin{array}{l}
\mathbf{a} \\
\mathbf{b}
\end{array}\right](\mathbf{Z})=\sum_{\left(l_{1}, l_{2}\right) \in \mathbb{Z}^{2}} q^{\frac{1}{2}\left(l_{1}+\frac{a_{1}}{2}\right)^{2}} r^{\left(l_{1}+\frac{a_{1}}{2}\right)\left(l_{2}+\frac{a_{2}}{2}\right)} s^{\frac{1}{2}\left(l_{2}+\frac{a_{2}}{2}\right)^{2}} e^{i \pi\left(l_{1} b_{1}+l_{2} b_{2}\right)},
$$

where $\mathbf{a}=\left(\begin{array}{l}a_{1} \\ a_{2}\end{array}\right), \mathbf{b}=\left(\begin{array}{l}b_{1} \\ b_{2}\end{array}\right)$, and $\mathbf{Z}=\left(\begin{array}{ll}z_{1} & z_{2} \\ z_{2} & z_{3}\end{array}\right) \in \mathbb{H}_{2}$. Further, we have defined $q=\exp \left(2 \pi i z_{1}\right), r=\exp \left(2 \pi i z_{2}\right)$ and $s=\exp \left(2 \pi i z_{3}\right)$. The constants $\left(a_{1}, a_{2}, b_{1}, b_{2}\right)$ take values $(0,1)$. Thus there are sixteen genus-two theta constants. The even theta constants are those for which $\mathbf{a}^{\mathrm{T}} \mathbf{b}=0 \bmod 2$. There are ten such theta constants for which we list the values of $\mathbf{a}$ and $\mathbf{b}$ : 


\begin{tabular}{|c|c|c|c|c|c|c|c|c|c|c|}
\hline$m$ & 0 & 1 & 2 & 3 & 4 & 5 & 6 & 7 & 8 & 9 \\
\hline$\left(\begin{array}{l}\mathbf{a} \\
\mathbf{b}\end{array}\right)$ & $\left(\begin{array}{l}0 \\
0 \\
0 \\
0\end{array}\right)$ & $\left(\begin{array}{l}0 \\
1 \\
0 \\
0\end{array}\right)$ & $\left(\begin{array}{l}1 \\
0 \\
0 \\
0\end{array}\right)$ & $\left(\begin{array}{l}1 \\
1 \\
0 \\
0\end{array}\right)$ & $\left(\begin{array}{l}0 \\
0 \\
0 \\
1\end{array}\right)$ & $\left(\begin{array}{l}1 \\
0 \\
0 \\
1\end{array}\right)$ & $\left(\begin{array}{l}0 \\
0 \\
1 \\
0\end{array}\right)$ & $\left(\begin{array}{l}0 \\
1 \\
1 \\
0\end{array}\right)$ & $\left(\begin{array}{l}0 \\
0 \\
1 \\
1\end{array}\right)$ & $\left(\begin{array}{l}1 \\
1 \\
1 \\
1\end{array}\right)$ \\
\hline
\end{tabular}

We will refer to the above ten theta constants as $\theta_{m}(\mathbf{Z})$ with $m=0,1, \ldots, 9$ representing the ten values of $\mathbf{a}$ and $\mathbf{b}$ as defined in the above table.

The modular functions $\Delta_{5}(\mathbf{Z})$ and $\Delta_{3}(\mathbf{Z})$ can be written out in terms of the even theta constants $[4,49]$. One finds

$$
\begin{aligned}
& \Delta_{5}(\mathbf{Z})=\frac{1}{64} \prod_{m=0}^{9} \theta_{m}(\mathbf{Z}) \\
& \Delta_{3}(\mathbf{Z})=\frac{1}{64} \theta_{2}(\mathbf{Z}) \prod_{m=1 \bmod 2} \theta_{m}(\mathbf{Z}) .
\end{aligned}
$$

Following ref. [49], the formula for $\Delta_{3}(\mathbf{Z})$ was constructed experimentally by looking for a product of six even theta constants that had the correct series expansion. This is also in agreement with the expression for $\Phi_{6}(\mathbf{Z})$ given in ref. [35] in terms of theta constants.

Let us define $\tilde{\Delta}_{3}(\mathbf{Z})$ to be the square-root of $\tilde{\Phi}_{6}(\mathbf{Z})$. One may wonder if it can also be written as the product of six genus-two theta constants. Keeping in mind that the leading term will go as $q^{1 / 4} r^{1 / 2} s^{1 / 2}$, we find that the following combination achieves this (to the orders that we have verified):

$$
\tilde{\Delta}_{3}(\mathbf{Z})=\frac{1}{16} \theta_{1}(\mathbf{Z}) \theta_{3}(\mathbf{Z}) \theta_{6}(\mathbf{Z}) \theta_{7}(\mathbf{Z}) \theta_{8}(\mathbf{Z}) \theta_{9}(\mathbf{Z})
$$

squares to given $\tilde{\Phi}_{6}(\mathbf{Z})$.

\section{B Jacobi cusp forms}

\section{B.1 Basic group theory}

The group $S p(2, \mathbb{Z})$ is the set of $4 \times 4$ matrices written in terms of four $2 \times 2$ matrices $A, B, C, D$ as 18

$$
M=\left(\begin{array}{ll}
A & B \\
C & D
\end{array}\right)
$$

satisfying $A B^{T}=B A^{T}, C D^{T}=D C^{T}$ and $A D^{T}-B C^{T}=I$. The congruence subgroup $\widehat{G}_{0}(N)$ of $S p(2, \mathbb{Z})$ is given by the set of matrices such that $C=0$ $\bmod N$. This group acts naturally on the Siegel upper half space, $\mathbb{H}_{2}$, as

$$
\mathbf{Z}=\left(\begin{array}{ll}
z_{1} & z_{2} \\
z_{2} & z_{3}
\end{array}\right) \longmapsto M \cdot \mathbf{Z} \equiv(A \mathbf{Z}+B)(C \mathbf{Z}+D)^{-1}
$$

\footnotetext{
${ }^{18}$ This section is based on the book by Eichler and Zagier [50].
} 
The Jacobi group $\Gamma^{J}=S L(2, \mathbb{Z}) \ltimes H(\mathbb{Z})$ is the sub-group of $S p(2, \mathbb{Z})$ that preserves the one-dimensional cusp $z_{3}=i \infty$. The $S L(2, \mathbb{Z})$ is generated by the embedding of $\left(\begin{array}{ll}a & b \\ c & d\end{array}\right) \in S L(2, \mathbb{Z})$ in $S p(2, \mathbb{Z})$

$$
g_{1}(a, b ; c, d) \equiv\left(\begin{array}{cccc}
a & 0 & b & 0 \\
0 & 1 & 0 & 0 \\
c & 0 & d & 0 \\
0 & 0 & 0 & 1
\end{array}\right)
$$

The above matrix acts on $\mathbb{H}_{2}$ as

$$
\left(z_{1}, z_{2}, z_{3}\right) \longrightarrow\left(\frac{a z_{1}+b}{c z_{1}+d}, \frac{z_{2}}{c z_{1}+d}, z_{3}-\frac{c z_{2}^{2}}{c z_{1}+d}\right)
$$

with $\operatorname{det}(C \mathbf{Z}+D)=\left(c z_{1}+d\right)$. The Heisenberg group, $H(\mathbb{Z})$, is generated by $S p(2, \mathbb{Z})$ matrices of the form

$$
g_{2}(\lambda, \mu, \kappa) \equiv\left(\begin{array}{cccc}
1 & 0 & 0 & \mu \\
\lambda & 1 & \mu & \kappa \\
0 & 0 & 1 & -\lambda \\
0 & 0 & 0 & 1
\end{array}\right) \quad \text { with } \lambda, \mu, \kappa \in \mathbb{Z}
$$

The above matrix acts on $\mathbb{H}_{2}$ as

$$
\left(z_{1}, z_{2}, z_{3}\right) \longrightarrow\left(z_{1}, \lambda z_{1}+z_{2}+\mu, z_{3}+\lambda^{2} z_{1}+2 \lambda z_{2}+\lambda \mu\right)
$$

with $\operatorname{det}(C \mathbf{Z}+D)=1$. It is easy to see that $\Gamma^{J}$ preserves the one-dimensional cusp at $\operatorname{Im}\left(z_{3}\right)=\infty$.

The full group $S p(2, \mathbb{Z})$ is generated by adding the exchange element to the group $\Gamma^{J}$.

$$
g_{3} \equiv\left(\begin{array}{cccc}
0 & 1 & 0 & 0 \\
1 & 0 & 0 & 0 \\
0 & 0 & 0 & 1 \\
0 & 0 & 1 & 0
\end{array}\right)
$$

This acts on $\mathbb{H}_{2}$ exchanging $z_{1} \leftrightarrow z_{3}$. The subgroup $\widehat{G}_{0}(N)$ is generated by considering the same three sets of matrices with the additional condition that $\left(\begin{array}{ll}a & b \\ c & d\end{array}\right) \in \Gamma_{0}(N)$ i.e., $c=0 \bmod N$ in Eq. (B.2). Further, we will call the corresponding Jacobi group $\Gamma_{0}(N)^{J}$.

\section{B.2 Weak Jacobi forms}

A Siegel modular form of weight $k$ and character $v$ with respect to $S p(2, \mathbb{Z})$ is a holomorphic function $F: \mathbb{H}_{2} \rightarrow \mathbb{C}$ satisfying

$$
F(M \cdot \mathbf{Z})=v(M) \operatorname{det}(C \mathbf{Z}+D)^{k} F(\mathbf{Z}),
$$


for all $\mathbb{Z} \in \mathbb{H}_{2}$ and $M \in S p(2, \mathbb{Z})$. In the above definition, one can replace $S p(2, \mathbb{Z})$ by any of its sub-groups such as $\Gamma^{J}$ or $\widehat{G}_{0}(N)$.

A holomorphic function

$$
\phi_{k, t}\left(z_{1}, z_{2}\right): \quad \mathbb{H}_{1} \times \mathbb{C} \rightarrow \mathbb{C}
$$

is called a Jacobi form of weight $k$ and index $t \in \frac{1}{2} \mathbb{Z}$, if

1. The function

$$
\tilde{\phi}_{k}(\mathbf{Z})=\exp \left(2 \pi i t z_{3}\right) \phi_{k, t}\left(z_{1}, z_{2}\right)
$$

on $\mathbb{H}_{2}$ is a modular form of weight $k$ with respect to the Jacobi group $\Gamma^{J} \subset$ $\operatorname{Sp}(2, \mathbb{Z})$.

2. It has a Fourier expansion

$$
\phi_{k, t}\left(z_{1}, z_{2}\right)=\sum_{\ell \in t+\mathbb{Z}} f(n, \ell) q^{n} r^{\ell}
$$

such that the Fourier coefficients, $f(n, \ell)=0$, unless $n \geq 0$ and $4 n t-\ell^{2} \geq 0$.

For weak Jacobi forms, the coefficients $f(n, \ell)$ are non-vanishing only when $n \geq 0$ relaxing the condition involving $\left(4 n t-\ell^{2}\right)$. Jacobi forms of integer index were considered by Eichler and Zagier [50] and extended to half-integral indices by Gritsenko [45].

The elliptic genus of Calabi-Yau manifolds are weak Jacobi forms. Examples include:

$$
\begin{aligned}
\phi_{-2,1}\left(z_{1}, z_{2}\right) & =\mathcal{E}_{\text {st } \times T^{2}}\left(z_{1}, z_{2}\right)=\left(\frac{i \vartheta_{1}\left(z_{1}, z_{2}\right)}{\eta^{3}\left(z_{1}\right)}\right)^{2} \\
\phi_{0,1}\left(z_{1}, z_{2}\right) & =\mathcal{E}_{K 3}\left(z_{1}, z_{2}\right)=8 \sum_{i=2}^{4}\left(\frac{\vartheta_{i}\left(z_{1}, z_{2}\right)}{\vartheta_{i}\left(z_{1}, 0\right)}\right)^{2}
\end{aligned}
$$

We will see the appearance of weight zero Jacobi forms of the group $\Gamma_{0}(N)^{J}$ in writing product representations for the modular form $\Phi_{k}(\mathbf{Z})$.

$$
\phi_{0,1}^{(N)}(\tau, z)=\frac{2 N}{N+1} \alpha^{(N)}(\tau) \phi_{-2,1}(\tau, z)+\frac{1}{N+1} \phi_{0,1}(\tau, z),
$$

with $\alpha^{(N)}(\tau)=\frac{12 i}{\pi(N-1)} \partial_{\tau}[\ln \eta(\tau)-\ln \eta(N \tau)]$ is the Eisenstein series for $\Gamma_{0}(N)$. 
The Fourier expansion for $\phi_{0,1}^{(N)}$ at the cusp at $i \infty$

$$
\begin{aligned}
& \phi_{0,1}^{(2)}(\tau, z)=\left(2 r+4+\frac{2}{r}\right)+\left(4 r^{2}-8+\frac{4}{r^{2}}\right) q+O\left(q^{2}\right) \\
& \phi_{0,1}^{(3)}(\tau, z)=\left(2 r+2+\frac{2}{r}\right)+\left(2 r^{2}-2 r-\frac{2}{r}+\frac{2}{r^{2}}\right) q+O\left(q^{2}\right) \\
& \phi_{0,1}^{(5)}(\tau, z)=\left(2 r+\frac{2}{r}\right)+\left(2 r-4+\frac{2}{r}\right) q+O\left(q^{2}\right) .
\end{aligned}
$$

and about the cusp at 0 is

$$
\begin{aligned}
\phi_{0,1}^{(2)}=8 & +\left(-\frac{16}{r}+32-16 r\right) q^{1 / 2}+\left(\frac{8}{r^{2}}-\frac{64}{r}+112-64 r+8 r^{2}\right) q+O\left(q^{3 / 2}\right) \\
\phi_{0,1}^{(3)}=6 & +\left(-\frac{6}{r}+12-6 r\right) q^{1 / 3}+\left(-\frac{18}{r}+36-18 r\right) q^{2 / 3} \\
& +\left(\frac{6}{r^{2}}-\frac{42}{r}+72-42 r+6 r^{2}\right) q+O\left(q^{4 / 3}\right) \\
\phi_{0,1}^{(5)}= & +\left(-\frac{2}{r}+4-2 r\right) q^{1 / 3}+\left(-\frac{6}{r}+12-6 r\right) q^{2 / 5}+\left(-\frac{8}{r}+16-8 r\right) q^{3 / 5} \\
& +\left(-\frac{14}{r}+28-14 r\right) q^{4 / 5}+\left(\frac{4}{r^{2}}-\frac{26}{r}+44-26 r+4 r^{2}\right) q+O\left(q^{6 / 5}\right)
\end{aligned}
$$

\section{B.3 Additive lift of Jacobi forms with integer index}

Given a Jacobi form of weight $k$ and index 1, Maaß constructed a Siegel modular form of weight $k$ leading to an explicit formula [51]

$$
\Phi_{k}(\mathbf{Z}) \equiv \sum_{(n, \ell, m)>0} \sum_{d \mid(n, \ell, m)} d^{k-1} f\left(\frac{n m}{d^{2}}, \frac{\ell}{d}\right) q^{n} r^{\ell} s^{m} .
$$

This procedure is known as the arithmetic or additive lift of the Jacobi form. It is known that the ring of Siegel modular forms is generated by four modular forms with weights 4, 6, 10 and 12 . For instance, the weight 10 modular form, $\Phi_{10}(\mathbf{Z})$, is generated by the Jacobi form of weight 10 and index 1

$$
\phi_{10,1}\left(z_{1}, z_{2}\right)=\theta_{1}\left(z_{1}, z_{2}\right)^{2} \eta\left(z_{1}\right)^{18} \text {. }
$$

Given a weight $k$, index 1 Jacobi form of the subgroup $\Gamma_{0}(N)^{J}$, one has a similar formula leading to a level- $N$ Siegel modular form i.e, a modular form of $\widehat{G}_{0}(N)$, is given by $[54-56]$

$$
\Phi_{k}(\mathbf{Z}) \equiv \sum_{(n, \ell, m)>0} \sum_{d \mid(n, \ell, m)} \chi(d) d^{k-1} f\left(\frac{n m}{d^{2}}, \frac{\ell}{d}\right) q^{n} r^{\ell} s^{m}
$$


where $\chi(d)$ is a real Dirichlet character [52] modulo $N$. When $\chi(d)$ is trivial, i.e.,

$$
\chi(d)= \begin{cases}0 & \text { if }(d, N) \neq 1 \\ 1 & \text { otherwise }\end{cases}
$$

we obtain a level $N$ Siegel modular form. For levels $N=2,3,5$, Jatkar and Sen have constructed modular forms of weight $k=6,4,2$ respectively as additive lifts of the weight $k$ Jacobi forms [9]:

$$
\phi_{k, 1}\left(z_{1}, z_{2}\right)=\theta_{1}\left(z_{1}, z_{2}\right)^{2} \eta\left(z_{1}\right)^{k-4} \eta\left(N z_{1}\right)^{k+2} .
$$

When the Jacobi form is one with character, one sees the appearance of a nontrivial Dirichlet character and the Siegel modular form obtained from the additive lift is one with character. At level $N=7$, Jatkar and Sen have constructed a modular form of weight 1 with character [9]. In this case, one does indeed see the appearance of a non-trivial real Dirichlet character in Eq. (B.15) given above.

\section{The additive lift with character at level $N$}

Let $\Delta_{k}(\mathbf{Z})$ be a Siegel modular form of $\widehat{G}_{0}(N) \subset S p(2, \mathbb{Z})$ with character $v^{\Gamma}$ i.e.,

$$
\Delta_{k}(M \cdot \mathbf{Z})=v^{\Gamma}(M) \operatorname{det}(C \mathbf{Z}+D)^{k} \Delta_{k}(\mathbf{Z}),
$$

where $v^{\Gamma}(M)$ is the unique non-trivial real linear character of $S p(2, \mathbb{Z})[53]$ and $M=\left(\begin{array}{cc}A & B \\ C & D\end{array}\right) \in \widehat{G}_{0}(N)$. An explicit expression for $v^{\Gamma}(M)$ is $[57]$

$$
\begin{gathered}
v^{\Gamma}\left(\begin{array}{cc}
0 & -I_{2} \\
I_{2} & 0
\end{array}\right)=1, v^{\Gamma}\left(\begin{array}{cc}
I_{2} & B \\
0 & I_{2}
\end{array}\right)=(-1)^{b_{1}+b_{2}+b}, \\
v^{\Gamma}\left(\begin{array}{cc}
U^{T} & 0 \\
0 & U^{1}
\end{array}\right)=(-1)^{\left(1+u_{0}+u_{2}\right)\left(1+u_{1}+u_{3}\right)+u_{0} u_{2}},
\end{gathered}
$$

where $I_{2}$ is the $2 \times 2$ identity matrix, $B=\left(\begin{array}{cc}b_{1} & b \\ b & b_{2}\end{array}\right)$ and $U=\left(\begin{array}{ll}u_{0} & u_{3} \\ u_{1} & u_{2}\end{array}\right)$ is a uni-modular matrix. The embedding of $S L(2, \mathbb{Z})$ in $S p(2 \mathbb{Z})$ given in Eq. (B.2) induces a character for $S L(2, \mathbb{Z})$. Given a $S L(2, \mathbb{Z})$ matrix $M$, let $M^{*}$ denote the corresponding $S p(2, \mathbb{Z})$ matrix as determined by Eq. (B.2), one has

$$
w^{\Gamma}(M) \equiv v^{\Gamma}\left(M^{*}\right)
$$

where $w^{\Gamma}(M)$ is the $S L(2, \mathbb{Z})$ character induced by the character of $S p(2, \mathbb{Z})$. It is useful to note that $w^{\Gamma}(S)=1$ when $S=I_{2} \bmod 2$.

Such a modular form admits a Fourier-Jacobi expansion of the form

$$
\Delta_{k}(\mathbf{Z})=\sum_{m=1} \psi_{k, m / 2}\left(z_{1}, z_{2}\right) s^{m / 2},
$$


where $\psi_{k, m / 2}\left(z_{1}, z_{2}\right)$ is a Jacobi form of weight $k$ and index $m / 2$ and character $w^{\Gamma}$ (induced by $v^{\Gamma}$ ). The condition that the exponent in the Fourier expansion be half-integral follows from the behavior of the character. Similarly, one also has the following transformations of $\psi_{k, m / 2}\left(z_{1}, z_{2}\right)$

$$
\begin{aligned}
\psi_{k, m / 2}\left(z_{1}, z_{2}+h\right) & =(-1)^{h} \psi_{k, m / 2}\left(z_{1}, z_{2}\right), \\
\psi_{k, m / 2}\left(z_{1}, \lambda z_{1}+z_{2}\right) & =(-1)^{\lambda} \psi_{k, m / 2}\left(z_{1}, z_{2}\right) .
\end{aligned}
$$

Thus, one sees that Fourier expansion of the Jacobi form about the cusp at $i \infty$ has half-integral exponents. An important point to note is that the weight $k$ must be odd else the modular form vanishes [57].

\section{C.1 Additive lift of Jacobi forms with half-integer index}

So far we have only considered examples of modular forms obtained from the lift of Jacobi forms with integral index. We will now consider examples with half-integral index as they appear in the denominator for the the GKM algebras, $\mathcal{G}_{N}$ discussed in the main body of the paper. The simplest example is given by the Jacobi theta function, $\vartheta_{1}\left(z_{1}, z_{2}\right)$. It is a holomorphic Jacobi form of weight $1 / 2$ and index $1 / 2$ with character. This Jacobi form appears as the denominator formula of the affine Kac-Moody algebra, $\widehat{A}_{1}^{(1)}$.

The Jacobi form of weight 5 and index $1 / 2$

$$
\psi_{5,1 / 2}\left(z_{1}, z_{2}\right)=\vartheta_{1}\left(z_{1}, z_{2}\right) \eta\left(z_{1}\right)^{9},
$$

generates the Siegel modular form with character, $\Delta_{5}(\mathbf{Z})$ via the additive lift. The Fourier expansion of the Jacobi form now involves half-integral exponents. One has

$$
\psi_{5,1 / 2}\left(z_{1}, z_{2}\right)=\sum_{n, \ell=1 \bmod 2} g(n, \ell) q^{n / 2} r^{\ell / 2},
$$

with $g(n, \ell)=0$ unless $4 n-\ell^{2} \geq 0$. The modular form $\Delta_{5}(\mathbf{Z})$ has the following expansion [57]

$$
\Delta_{5}(\mathbf{Z})=\sum_{(n, \ell, m)>0} \sum_{d \mid(n, \ell, m)} d^{k-1} g\left(\frac{n m}{d^{2}}, \frac{\ell}{d}\right) q^{n / 2} r^{\ell / 2} s^{m / 2} .
$$

Notice the similarity with the Maaß formula given in Eq. (B.15) with half-integral powers of $q, r$ and $s$ appearing where integral powers appeared. Gritsenko and Nikulin have shown that this modular form appears as the denominator formula of a GKM superalgebra.

$\Delta_{5}(\mathbf{Z})$ is a modular form with character under the full modular group, $S p(2, \mathbb{Z})$. It transforms as

$$
\Delta_{5}(M \cdot \mathbf{Z})=v^{\Gamma}(M)(C \mathbf{Z}+D)^{5} \Delta_{5}(\mathbf{Z})
$$


We will now generalize the additive lift (C.10) to higher levels. In particular, we construct additive lifts of the Jacobi forms of index $1 / 2$ given in Eq. (4.2).

\section{C.2 Hecke operators and the additive lift}

Given a index-half Jacobi form $\psi_{k, 1 / 2}(\tau, z)$ with Fourier expansion

$$
\psi_{k, 1 / 2}(\tau, z)=\sum_{n, \ell} g(n, \ell) q^{n / 2} r^{\ell / 2}
$$

we construct a Jacobi form of the same weight and index $m / 2$ for odd $m$ by the following averaging procedure. This generalizes and closely follows the procedure due to Maaß [57] for Jacobi forms of index half to higher levels.

Let $X_{m}$ be the group of $2 \times 2$ matrices $M=\left(\begin{array}{cc}\alpha & \beta \\ \gamma & \delta\end{array}\right)$ with integral entries such that (i) $\operatorname{det}(M)=m(m=1 \bmod 2)$; (ii) $M=I_{2} \bmod 2$; (iii) $\gamma=0 \bmod N$ and (iv) $(\alpha, N)=1$. Note that $X_{1}$ is a subgroup of $\Gamma_{0}(N)$ for which $w^{\Gamma}(M)=v^{\Gamma}\left(M^{*}\right)=1$. We will show that the following is a Jacobi form of weight $k$ and index $m / 2$ :

$$
\psi_{k, m / 2}(\tau, z) \equiv(m)^{k-1} \sum_{\left(\begin{array}{cc}
\alpha & \beta \\
\gamma & \delta
\end{array}\right) \in X_{1} \backslash X_{m}}(\gamma \tau+\delta)^{-k} e^{\pi i m \frac{\gamma z^{2}}{\gamma \tau+\delta}} \psi_{k, 1 / 2}\left(\frac{\alpha \tau+\beta}{\gamma \tau+\delta}, \frac{m z}{\gamma \tau+\delta}\right)
$$

Claim: Consider the coset $X_{1} \backslash X_{m}$. The coset can be represented by the elements

$$
\left(\begin{array}{cc}
\alpha & 2 \beta \\
0 & \delta
\end{array}\right) \text {, with } \alpha \delta=m, \beta=0,1, \ldots,(\delta-1),(\alpha, N)=1 \text { and } \alpha>0 \text {. }
$$

The proof is along lines similar to the one given by Jatkar-Sen $[9$, see appendix A] and will not be repeated here. Using the above representation of the coset $X_{1} \backslash X_{m}$ given above, we can rewrite the equation defining $\psi_{k, m / 2}(\tau, z)$ as follows:

$$
\begin{aligned}
\psi_{k, m / 2}(\tau, z) & =(m)^{k-1} \sum_{\substack{\alpha \delta=m \\
(\alpha, N)=1 \\
\alpha>0}} \delta^{-k} \sum_{\beta=0}^{\delta-1} \psi_{k, 1 / 2}\left(\frac{\alpha \tau+2 \beta}{\delta}, \frac{m z}{\delta}\right) \\
& =(m)^{k-1} \sum_{\substack{\alpha \delta=m \\
(\alpha, N)=1 \\
\alpha>0}} \delta^{-k} \sum_{\beta=0}^{\delta-1} \sum_{n, \ell} g(n, \ell) e^{i \pi n \frac{\alpha \tau+2 \beta}{\delta}} e^{i \pi \ell \frac{m z}{\delta}} \\
& =(m)^{k-1} \sum_{\substack{\alpha \delta=m \\
(\alpha, N)=1 \\
\alpha>0}} \delta^{-k+1} \sum_{\substack{n \mid \ell \\
\delta n \alpha}} g(n, \ell) e^{\frac{i \pi n \alpha \tau}{\delta}} e^{\frac{i \pi \ell m z}{\delta}} \\
& =\sum_{n^{\prime}, \ell^{\prime}} \sum_{\substack{\alpha \mid\left(n^{\prime}, \ell^{\prime}, m\right) \\
\alpha>0}} \chi(\alpha) \alpha^{k-1} g\left(\frac{n^{\prime} m}{\alpha^{2}}, \frac{\ell^{\prime}}{\alpha}\right) e^{i \pi n^{\prime} \tau} e^{i \pi \ell^{\prime} z},
\end{aligned}
$$


where $\chi(\alpha)$ is the trivial Dirichlet character modulo $N$ which implements the condition $(\alpha, N)=1$. In obtaining the last line, we have defined $n^{\prime}=n \alpha / \delta$ and $\ell^{\prime}=\ell \alpha$ and replaced $\delta$ by $m / \alpha$.

Modularity: We now prove that $\psi_{k, m / 2}(\tau, z)$ constructed by the averaging procedure is a Jacobi form with character. Let us first consider the transformation under $M_{1}=\left(\begin{array}{ll}a & b \\ c & d\end{array}\right) \in \Gamma_{0}(N)$. The proof follows along the lines of Maaß [57] (and Jatkar-Sen [9] for the modifications to account for the level.). We only repeat the crucial argument. Given any matrix $S \in X_{1} \backslash X_{m}$, one has $S M_{1}=\tilde{M}_{1} \tilde{S}$ where $\tilde{M}_{1}=\left(\begin{array}{cc}\tilde{a} & \tilde{b} \\ \tilde{c} & \tilde{d}\end{array}\right) \in \Gamma_{0}(N)$ and $\tilde{S}$ is another matrix in $X_{1} \backslash X_{m}$. In other words, averaging over all $S$ can be replaced by averaging over $\tilde{S}$ with $\tilde{M}_{1}$ taking the place of $M_{1}$. In particular, one has

$$
\psi_{k, 1 / 2}\left(\frac{\tilde{a} \tau+\tilde{b}}{\tilde{c} \tau+\tilde{d}}, \frac{z}{\tilde{c} \tau+\tilde{d}}\right) e^{-i \pi \frac{\tilde{c} z^{2}}{\tilde{c} \tau+\tilde{d}}}=w^{\Gamma}\left(\tilde{M}_{1}\right)(\tilde{c} \tau+\tilde{d})^{k} \psi_{k, 1 / 2}(\tau, z) .
$$

Since $w^{\Gamma}\left(S M_{1}\right)=w^{\Gamma}\left(\tilde{M}_{1} \tilde{S}\right)$ and $w^{\Gamma}(S)=w^{\Gamma}(\tilde{S})=1$, we see that $w^{\Gamma}\left(M_{1}\right)=$ $w^{\Gamma}\left(\tilde{M}_{1}\right)$ leading to the required result.

$$
\psi_{k, m / 2}\left(\frac{a \tau+b}{c \tau+d}, \frac{z}{c \tau+d}\right) e^{-i \pi m \frac{c z^{2}}{c \tau+c}}=w^{\Gamma}\left(M_{1}\right)(c \tau+d)^{k} \psi_{k, m / 2}(\tau, z) .
$$

The transformation under the other generators of the Jacobi group, $\Gamma_{0}(N)^{J}$, follow in an elementary fashion. Thus, we see that $\psi_{k, m / 2}$ is a Jacobi form of weight $k$ and index $m / 2$ for odd $m$.

One thus sees that

$$
\begin{aligned}
\Delta_{k}(\mathbf{Z}) & =\sum_{m=1 \bmod 2 ; m>0} \psi_{k, m / 2}\left(z_{1}, z_{2}\right) e^{i \pi m z_{3}} \\
& =\sum_{(n, \ell, m)>0} \sum_{\substack{\alpha \mid(n, \ell, m) \\
\alpha>0}} \chi(\alpha) \alpha^{k-1} g\left(\frac{n m}{\alpha^{2}}, \frac{\ell}{\alpha}\right) q^{n / 2} r^{\ell / 2} s^{m / 2}
\end{aligned}
$$

is a modular form of $\widehat{G}_{0}(N)$ with (odd) weight $k$ and character $v^{\Gamma}$. When the seed Jacobi form is a weak one, then the above sum is not convergent and one obtains a meromorphic modular form [56]. This is indeed the case in our example for $N=5$.

When $N=3$, the weight of the modular form we have constructed is even as $k=2$. However, the seed Jacobi form, $\psi_{2,1 / 2}(\tau, z)$, transforms with character $w^{\gamma} w^{\psi}$ and not as in Eq. (C.19), thus evading the restriction on $k$ being odd. Taking into account the additional character, $w^{\psi}$, one obtains a formula similar to Eq. (C.21) but with $\chi^{\psi}(\alpha)$ as defined in Eq. (4.5) replacing $\chi(\alpha)$ :

$$
\Delta_{2}(\mathbf{Z})=\sum_{(n, \ell, m)>0} \sum_{\substack{\alpha \mid(n, \ell, m) \\ \alpha>0}} \chi^{\psi}(\alpha) \alpha^{k-1} g\left(\frac{n m}{\alpha^{2}}, \frac{\ell}{\alpha}\right) q^{n / 2} r^{\ell / 2} s^{m / 2}
$$




\section{Explicit formulae for $\Delta_{k / 2}(\mathbf{Z})$}

We note that $\Delta_{k / 2}(\mathbf{Z})$ is symmetric under the exchange $z_{1} \leftrightarrow z_{3}$ and is antisymmetric under $z_{2} \rightarrow-z_{2}$ for all values of $k$.

$$
\begin{aligned}
& \Delta_{5}=\left(-\frac{1}{\sqrt{r}}+\sqrt{r}\right) \sqrt{q} \sqrt{s}+\left(\frac{9}{r^{\frac{5}{2}}}-\frac{93}{r^{\frac{3}{2}}}+\frac{90}{\sqrt{r}}-90 \sqrt{r}+93 r^{\frac{3}{2}}-9 r^{\frac{5}{2}}\right) q^{\frac{3}{2}} s^{\frac{3}{2}} \\
& +\left(r^{-\frac{3}{2}}+\frac{9}{\sqrt{r}}-9 \sqrt{r}-r^{\frac{3}{2}}\right)\left(q^{\frac{3}{2}} \sqrt{s}+\sqrt{q} s^{\frac{3}{2}}\right) \\
& +\left(\frac{-9}{r^{\frac{3}{2}}}-\frac{27}{\sqrt{r}}+27 \sqrt{r}+9 r^{\frac{3}{2}}\right)\left(q^{\frac{5}{2}} \sqrt{s}+\sqrt{q} s^{\frac{5}{2}}\right) \\
& +\left(-r^{-\frac{5}{2}}+\frac{27}{r^{\frac{3}{2}}}+\frac{12}{\sqrt{r}}-12 \sqrt{r}-27 r^{\frac{3}{2}}+r^{\frac{5}{2}}\right)\left(q^{\frac{7}{2}} \sqrt{s}+\sqrt{q} s^{\frac{7}{2}}\right) \\
& +\left(\frac{9}{r^{\frac{5}{2}}}-\frac{12}{r^{\frac{3}{2}}}+\frac{90}{\sqrt{r}}-90 \sqrt{r}+12 r^{\frac{3}{2}}-9 r^{\frac{5}{2}}\right)\left(q^{\frac{9}{2}} \sqrt{s}+\sqrt{q} s^{\frac{9}{2}}\right) \\
& +\left(\frac{-27}{r^{\frac{5}{2}}}-\frac{90}{r^{\frac{3}{2}}}-\frac{135}{\sqrt{r}}+135 \sqrt{r}+90 r^{\frac{3}{2}}+27 r^{\frac{5}{2}}\right)\left(q^{\frac{11}{2}} \sqrt{s}+\sqrt{q} s^{\frac{11}{2}}\right) \\
& +\left(r^{-\frac{7}{2}}+\frac{12}{r^{\frac{5}{2}}}+\frac{135}{r^{\frac{3}{2}}}-\frac{54}{\sqrt{r}}+54 \sqrt{r}-135 r^{\frac{3}{2}}-12 r^{\frac{5}{2}}-r^{\frac{7}{2}}\right)\left(q^{\frac{13}{2}} \sqrt{s}+\sqrt{q} s^{\frac{13}{2}}\right) \\
& \Delta_{3}=\left(-\frac{1}{\sqrt{r}}+\sqrt{r}\right) \sqrt{q} \sqrt{s}+\left(r^{-\frac{5}{2}}-\frac{5}{r^{\frac{3}{2}}}-\frac{6}{\sqrt{r}}+6 \sqrt{r}+5 r^{\frac{3}{2}}-r^{\frac{5}{2}}\right) q^{\frac{3}{2}} s^{\frac{3}{2}} \\
& +\left(r^{-\frac{3}{2}}+\frac{1}{\sqrt{r}}-\sqrt{r}-r^{\frac{3}{2}}\right)\left(q^{\frac{3}{2}} \sqrt{s}+\sqrt{q} s^{\frac{3}{2}}\right) \\
& +\left(-r^{-\frac{3}{2}}+\frac{5}{\sqrt{r}}-5 \sqrt{r}+r^{\frac{3}{2}}\right)\left(q^{\frac{5}{2}} \sqrt{s}+\sqrt{q} s^{\frac{5}{2}}\right) \\
& +\left(-r^{-\frac{5}{2}}-\frac{5}{r^{\frac{3}{2}}}-\frac{4}{\sqrt{r}}+4 \sqrt{r}+5 r^{\frac{3}{2}}+r^{\frac{5}{2}}\right)\left(q^{\frac{7}{2}} \sqrt{s}+\sqrt{q} s^{\frac{7}{2}}\right) \\
& +\left(r^{-\frac{5}{2}}+\frac{4}{r^{\frac{3}{2}}}-\frac{6}{\sqrt{r}}+6 \sqrt{r}-4 r^{\frac{3}{2}}-r^{\frac{5}{2}}\right)\left(q^{\frac{9}{2}} \sqrt{s}+\sqrt{q} s^{\frac{9}{2}}\right) \\
& +\left(\frac{5}{r^{\frac{5}{2}}}+\frac{6}{r^{\frac{3}{2}}}+\frac{1}{\sqrt{r}}-\sqrt{r}-6 r^{\frac{3}{2}}-5 r^{\frac{5}{2}}\right)\left(q^{\frac{11}{2}} \sqrt{s}+\sqrt{q} s^{\frac{11}{2}}\right) \\
& +\left(r^{-\frac{7}{2}}-\frac{4}{r^{\frac{5}{2}}}-r^{-\left(\frac{3}{2}\right)}-\frac{6}{\sqrt{r}}+6 \sqrt{r}+r^{\frac{3}{2}}+4 r^{\frac{5}{2}}-r^{\frac{7}{2}}\right)\left(q^{\frac{13}{2}} \sqrt{s}+\sqrt{q} s^{\frac{13}{2}}\right)
\end{aligned}
$$




$$
\begin{aligned}
\Delta_{2} & =\left(\sqrt{r}-\frac{1}{\sqrt{r}}\right) \sqrt{q} \sqrt{s}+\left(3 r^{3 / 2}-\frac{3}{r^{3 / 2}}\right) q^{3 / 2} s^{3 / 2}+\left(\frac{1}{r^{3 / 2}}-r^{3 / 2}\right)\left(\sqrt{s} q^{3 / 2}+s^{3 / 2} \sqrt{q}\right) \\
& +\left(r^{5 / 2}-3 \sqrt{r}+\frac{3}{\sqrt{r}}-\frac{1}{r^{5 / 2}}\right)\left(\sqrt{s} q^{7 / 2}+s^{7 / 2} \sqrt{q}\right)+\left(3 r^{3 / 2}-\frac{3}{r^{3 / 2}}\right)\left(\sqrt{s} q^{9 / 2}+s^{9 / 2} \sqrt{q}\right) \\
+ & \left(-r^{7 / 2}-3 r^{5 / 2}+\frac{3}{r^{5 / 2}}+\frac{1}{r^{7 / 2}}\right)\left(\sqrt{s} q^{13 / 2}+s^{13 / 2} \sqrt{q}\right) \\
+ & \left(3 r^{7 / 2}+5 \sqrt{r}-\frac{5}{\sqrt{r}}-\frac{3}{r^{7 / 2}}\right)\left(\sqrt{s} q^{19 / 2}+s^{19 / 2} \sqrt{q}\right) \\
\Delta_{1} & \left(\sqrt{r}-\frac{1}{\sqrt{r}}\right) \sqrt{q} \sqrt{s}+\left(-r^{3 / 2}+\sqrt{r}-\frac{1}{\sqrt{r}}+\frac{1}{r^{3 / 2}}\right)\left(\sqrt{s} q^{3 / 2}+s^{3 / 2} \sqrt{q}\right) \\
+ & \left(r^{5 / 2}-2 r^{3 / 2}+5 \sqrt{r}-\frac{5}{\sqrt{r}}+\frac{2}{r^{3 / 2}}-\frac{1}{r^{5 / 2}}\right) q^{3 / 2} s^{3 / 2} \\
+ & \left(-r^{3 / 2}+2 \sqrt{r}-\frac{2}{\sqrt{r}}+\frac{1}{r^{3 / 2}}\right)\left(\sqrt{s} q^{5 / 2}+s^{5 / 2} \sqrt{q}\right) \\
+ & \left(r^{5 / 2}-2 r^{3 / 2}+3 \sqrt{r}-\frac{3}{\sqrt{r}}+\frac{2}{r^{3 / 2}}-\frac{1}{r^{5 / 2}}\right)\left(\sqrt{s} q^{7 / 2}+s^{7 / 2} \sqrt{q}\right) \\
+ & \left(r^{5 / 2}-3 r^{3 / 2}+5 \sqrt{r}-\frac{5}{\sqrt{r}}+\frac{3}{r^{3 / 2}}-\frac{1}{r^{5 / 2}}\right)\left(\sqrt{s} q^{9 / 2}+s^{9 / 2} \sqrt{q}\right) \\
+ & \left(2 r^{5 / 2}-5 r^{3 / 2}+5 \sqrt{r}-\frac{5}{\sqrt{r}}+\frac{5}{r^{3 / 2}}-\frac{2}{r^{5 / 2}}\right)\left(\sqrt{s} q^{11 / 2}+s^{11 / 2} \sqrt{q}\right) \\
+ & \left(-r^{7 / 2}+3 r^{5 / 2}-5 r^{3 / 2}+9 \sqrt{r}-\frac{9}{\sqrt{r}}+\frac{5}{r^{3 / 2}}-\frac{3}{r^{5 / 2}}+\frac{1}{r^{7 / 2}}\right)\left(\sqrt{s} q^{13 / 2}+s^{13 / 2} \sqrt{q}\right) \\
+ &
\end{aligned}
$$

\section{D.1 Analyzing the modular forms}

We list some of the observations that can be made from the above expansions.

1. Using the expressions for the real simple roots, $\left(\delta_{1}, \delta_{2}, \delta_{3}\right)$ and their inner product with $\mathbf{Z}$, one sees that

$$
e^{-\pi i\left(\delta_{1}, \mathbf{Z}\right)}=q r, e^{-\pi i\left(\delta_{2}, \mathbf{Z}\right)}=r^{-1} \text { and } e^{-\pi i\left(\delta_{3}, \mathbf{Z}\right)}=s r .
$$

(Recall that $q=\exp \left(2 \pi i z_{1}\right), r=\exp \left(2 \pi i z_{2}\right)$ and $s=\exp \left(2 \pi i z_{3}\right)$.) Thus, one has $\exp (-\pi i(\rho, \mathbf{Z}))=q^{1 / 2} r^{1 / 2} s^{1 / 2}$. Further, one has the identification relating the root $\alpha[n, \ell, m]$ to $q^{n} r^{\ell} s^{m}$ :

$$
q^{n} r^{\ell} s^{m}=e^{-\pi i(\alpha[n, \ell, m], \mathbf{Z})},
$$


where the root $\alpha[n, \ell, m]=n \delta_{1}+(-l+m+n) \delta_{2}+m \delta_{3}$ has norm $\left(2 \ell^{2}-8 n m\right)$. The real simple roots are $(\alpha[1,1,0], \alpha[0,-1,0], \alpha[0,1,1])$ and the Weyl vector is $\rho=\alpha\left[\frac{1}{2}, \frac{1}{2}, \frac{1}{2}\right]$ in this notation.

2. In the expansion for $\Delta_{5}(\mathbf{Z})$, all terms (in the expansion given above) that arise with coefficient \pm 1 come from the sum side of the Lie algebra $\mathfrak{g}\left(A_{1, I I}\right)$. In other words, they arise by the action of all elements of the Weyl group generated by the three real simple roots. For instance, the terms arising from Weyl reflections associated with the simple real roots of $\mathcal{G}_{1}$ are

$$
\left(q^{3 / 2} r^{3 / 2} s^{1 / 2}, q^{1 / 2} r^{-1 / 2} s^{1 / 2}, q^{1 / 2} r^{3 / 2} s^{3 / 2}\right)=q^{1 / 2} r^{1 / 2} s^{1 / 2}\left(q r, r^{-1}, s r\right) .
$$

Note that we need to pull out an overall factor of $q^{1 / 2} r^{1 / 2} s^{1 / 2}$ in the sum side of the denominator formula to extract the roots.

3. The terms that appear in $\Delta_{5}(\mathbf{Z})$ with coefficient \pm 1 continue do so in the other modular forms $\Delta_{k / 2}(\mathbf{Z})$. This is consistent with the observation that the real roots are unaffected by the orbifolding. We have also verified that the terms involving imaginary roots related by Weyl reflections appear with the same multiplicity.

4. All the GKM superalgebras have an outer $S_{3}$ symmetry which permutes the three real simple roots. It is easy to see only the $\delta_{1} \leftrightarrow \delta_{3}$ (or equivalently the $q \leftrightarrow s$ ) symmetry in the $\Delta_{k / 2}(\mathbf{Z})$. A formal proof can be given by following Gritsenko and Nikulin's argument for $\mathcal{G}_{1}$ [4, see Prop. 2.1]. Their proof makes use of the non-trivial character $v^{\Gamma}$ appearing in the modular transform $\Delta_{5}(\mathbf{Z})$. All the modular forms, $\Delta_{k / 2}(\mathbf{Z})$, share the same character suitably restricted to the relevant subgroup of $S p(2, \mathbb{Z})$, the same proof goes through.

5. A practical check of the outer $S_{3}$ needs us to verify the $\delta_{1} \leftrightarrow \delta_{2}$ invariance of $\Delta_{k}(\mathbf{Z})$. One can show that under this exchange

$$
\alpha[n, \ell, m] \leftrightarrow \alpha[-\ell+m+n,-\ell+2 m, m] .
$$

For instance, the light-like root $\alpha[0,0,1]$ is mapped to another light-like root $\alpha[1,2,1]$. This relates the term $q^{1 / 2} r^{1 / 2} s^{3 / 2}$ to $q^{3 / 2} r^{5 / 2} s^{3 / 2}-$ both have multiplicity -9 in $\Delta_{5}(\mathbf{Z})$. These two terms appear with identical multiplicity -1 in $\Delta_{3}(\mathbf{Z}), 0$ in $\Delta_{2}(\mathbf{Z})$ and 1 in $\Delta_{1}(\mathbf{Z})$. A similar analysis has been carried out for other terms as well.

6. Another proof of the outer $S_{3}$ symmetry can be obtained by considering the product form of the modular form in Eq. (4.7). The multiplicities of 
positive roots is determined by two functions $c_{1}(n m, \ell)$ and $c_{2}(n m, \ell)$. These functions depend only on the combination $\left(4 n m-\ell^{2}\right)$ as they arise from the Fourier expansions of weak Jacobi forms. The action of the outer $S_{3}$ maps a positive root $\alpha[n, \ell, m]$, with norm $-2\left(4 n m-\ell^{2}\right)$, to another root with the same norm. Both these roots necessarily share the same multiplicity.

\section{References}

[1] A. Strominger and C. Vafa, "Microscopic Origin of the Bekenstein-Hawking Entropy," Phys. Lett. B 379 (1996) 99 arXiv:hep-th/9601029.

[2] M. J. Duff, J. T. Liu and J. Rahmfeld, "Four-Dimensional String-String-String Triality," Nucl. Phys. B 459 (1996) 125 arXiv:hep-th/9508094.

[3] R. Dijkgraaf, E. P. Verlinde and H. L. Verlinde, "Counting dyons in $\mathrm{N}=4$ string theory," Nucl. Phys. B 484 (1997) 543 arXiv:hep-th/9607026.

[4] V. A. Gritsenko, V. V. Nikulin, "Siegel Automorphic Form Corrections of Some Lorentzian Kac-Moody Lie Algebras," Amer. J. Math. 119 (1997) 181224 arXiv:alg-geom/9504006

[5] J. A. Harvey and G. W. Moore, "Algebras, BPS States, and Strings," Nucl. Phys. B 463 (1996) 315 arXiv:hep-th/9510182.

[6] J. A. Harvey and G. W. Moore, "On the algebras of BPS states," Commun. Math. Phys. 197 (1998) 489 arXiv:hep-th/9609017.

[7] M. C. N. Cheng and E. P. Verlinde, "Wall Crossing, Discrete Attractor Flow, and Borcherds Algebra," SIGMA 4 (2008) 068 arXiv:0806.2337 [hep-th].

[8] S. Chaudhuri, G. Hockney and J. D. Lykken, "Maximally Supersymmetric String Theories in $D<10$," Phys. Rev. Lett. 75 (1995) 2264 arXiv:hep-th/9505054.

[9] D. P. Jatkar and A. Sen, "Dyon spectrum in CHL models," JHEP 0604, 018 (2006) arXiv:hep-th/0510147.

[10] A. Sen, "Black Holes, Elementary Strings and Holomorphic Anomaly," JHEP 0507 (2005) 063 arXiv:hep-th/0502126.

[11] R. Borcherds, "The Monster Lie algebra," Advances In Mathematics 83, 30-47 (1990) 
[12] P. Niemann, "Some generalized Kac-Moody algebras with known root multiplicities," Mem. Amer. Math. Soc. 157 (2002), no. 746 arXiv:math.QA/0001029

[13] J. H. Schwarz and A. Sen, "Duality symmetric actions," Nucl. Phys. B 411 (1994) 35 arXiv:hep-th/9304154.

[14] S. Banerjee and A. Sen, "Duality Orbits, Dyon Spectrum and Gauge Theory Limit of Heterotic String Theory on $T^{6}$," JHEP 0803 (2008) 022 arXiv:0712.0043 [hep-th].

[15] M. Cvetic and D. Youm, "Dyonic BPS saturated black holes of heterotic string on a six torus," Phys. Rev. D 53 (1996) 584 arXiv:hep-th/9507090.

[16] W. Lerche and S. Stieberger, "1/4 BPS states and non-perturbative couplings in N = 4 string theories," Adv. Theor. Math. Phys. 3 (1999) 1539 arXiv:hep-th/9907133.

[17] J. R. David, D. P. Jatkar and A. Sen, "Product representation of dyon partition function in CHL models," JHEP 0606, 064 (2006) arXiv:hep-th/0602254.

[18] A. Dabholkar, F. Denef, G. W. Moore and B. Pioline, "Exact and asymptotic degeneracies of small black holes," JHEP 0508 (2005) 021 arXiv:hep-th/0502157.

[19] A. Dabholkar, F. Denef, G. W. Moore and B. Pioline, "Precision counting of small black holes," JHEP 0510 (2005) 096 arXiv:hep-th/0507014.

[20] A. Dabholkar, "Exact counting of black hole microstates," Phys. Rev. Lett. 94 (2005) 241301 arXiv:hep-th/0409148.

[21] A. Sen, "Entropy function for heterotic black holes," JHEP 0603 (2006) 008 arXiv:hep-th/0508042.

[22] A. Dabholkar and S. Nampuri, "Spectrum of Dyons and Black Holes in CHL orbifolds using Borcherds Lift," JHEP 0711, 077 (2007) arXiv:hep-th/0603066.

[23] J. R. David and A. Sen, "CHL dyons and statistical entropy function from D1-D5 system," JHEP 0611 (2006) 072 arXiv:hep-th/0605210.

[24] D. Shih, A. Strominger and X. Yin, "Recounting dyons in N = 4 string theory," JHEP 0610, 087 (2006) arXiv:hep-th/0505094. 
[25] R. Dijkgraaf, G. W. Moore, E. P. Verlinde and H. L. Verlinde, "Elliptic genera of symmetric products and second quantized strings," Commun. Math. Phys. 185 (1997) 197 arXiv:hep-th/9608096.

[26] V. G. Kac, "Infinite dimensional Lie algebras, 3rd ed.," Cambridge University Press, (1990).

[27] A. J. Feingold and I. B. Frenkel, "A hyperbolic Kac-Moody algebra and the theory of Siegel modular forms of genus 2', Math. Ann. 263 (1983), 87-144.

[28] A. J. Feingold and H. Nicolai, "Subalgebras of Hyperbolic Kac-Moody Algebras," arXiv:math/0303179.

[29] J. Lepowsky, S. Milne. "Lie Algebraic approaches to classical partition identities," Advances In Mathematics 29, (1978) 15-29.

[30] I. G. MacDonald, "Affine root systems and Dedekind's $\eta$-function," Invent. Math 15 (1972) 91-143.

[31] J. Lepowsky, H. Garland, "Lie Algebra homology and the Macdonald-Kac formulas," Invent. Math. 34, (1976) 37-76.

[32] R. Borcherds, "Generalized Kac-Moody algebras," Journal of Algebra 115, 501-512 (1988)

[33] R. Borcherds, "Monstrous Moonshine and Monstrous Lie Superalgebras," Invent. Math. 109, (1992) 405-444.

[34] E. Jurisich, "An exposition of Generalized Kac-Moody algebras." Contemporary Mathematics vol. 194, (1996) 121-159.

[35] H. Aoki and T. Ibukiyama, "Simple Graded Rings of Siegel Modular Forms, Differential Operators and Borcherds Products," Int. J. Math. 163 (2005), 249-279.

[36] A. Dabholkar and D. Gaiotto, "Spectrum of CHL dyons from genus-two partition function," JHEP 0712 (2007) 087 arXiv:hep-th/0612011.

[37] A. Sen, "Walls of Marginal Stability and Dyon Spectrum in N=4 Supersymmetric String Theories," JHEP 0705 (2007) 039 arXiv:hep-th/0702141.

[38] A. Sen, "Rare Decay Modes of Quarter BPS Dyons," JHEP 0710 (2007) 059 arXiv:0707.1563 [hep-th].

[39] A. Mukherjee, S. Mukhi and R. Nigam, "Dyon Death Eaters," JHEP 0710 (2007) 037 arXiv:0707.3035 [hep-th]. 
[40] A. Mukherjee, S. Mukhi and R. Nigam, "Kinematical Analogy for Marginal Dyon Decay," arXiv:0710.4533 [hep-th].

[41] M. C. N. Cheng and A. Dabholkar, "Borcherds-Kac-Moody Symmetry of $\mathcal{N}=4$ Dyons," arXiv:0809.4258 [hep-th].

[42] V. A. Gritsenko, V. V. Nikulin, "The Igusa Modular Forms and "the simples" Lorentzian Kac-Moody algebra," (Russian) Mat. Sb. 187 (1996), no. 11, 27-66; translation in Sb. Math. 187 (1996), no. 11, 1601-1641 arXiv:alg-geom/9603010.

[43] A. Garbagnati and A. Sarti, "Symplectic automorphisms of prime order on K3 surfaces," J. of Algebra 318 (2007) 323-350 arXiv:math/0603742.

[44] A. Garbagnati and A. Sarti, "Elliptic fibrations and symplectic automorphisms on K3 surfaces," arXiv:0801.3992v1 [math.AG].

[45] V. Gritsenko, "Elliptic genus of Calabi-Yau manifolds and Jacobi and Siegel modular forms," Algebra i Analiz 11 (1999), no. 5, 100-125; translation in St. Petersburg Math. J. 11 (2000), no. 5, 781-804 arXiv:math/9906190v1.

[46] R. Gopakumar and C. Vafa, "M-theory and topological strings. I," arXiv:hep-th/9809187.

[47] R. Gopakumar and C. Vafa, "M-theory and topological strings. II," arXiv:hep-th/9812127.

[48] B. Young (with an appendix by J. Bryan), "Generating functions for colored 3D Young diagrams and the Donaldson-Thomas invariants of orbifolds," arXiv:0802.2948[math.CO].

[49] S. Raghavan, "Cusp forms of degree 2 and weight 3," Math. Ann. 224, no. 2 (1976), 149-156.

[50] M. Eichler and D. Zagier, "The theory of Jacobi Forms," Birkhäuser (1985).

[51] H. Maaß,Inv. Math. 52 (1979) 95-104, Ibid. 53 (1979) 249-253, Ibid. 53 (179) 255-265.

[52] We found it useful to refer to the online encyclopedias: http://planetmath.org/encyclopedia/DirichletCharacter.html as well as http://en.wikipedia.org/wiki/Dirichlet_character. 
[53] I. Reiner, "Real linear characters of the symplectic modular group," Proc. Amer. Math. Soc. 6 (1955) pp. 987-990.

H. Maaß, "Die Multiplikatorsysteme zur Siegelschen Modulgruppe" Nachr. Akad. Wiss.Göttingen Math.-Phys. Kl. II (1964) 125-135.

[54] M. Manickam, B. Ramakrishnan, "On Saito-Kurokawa Descent for congruence subgroups," Manuscripta Math. 81 (1993) 161-182.

[55] M. Manickam, B. Ramakrishnan, "On Saito-Kurokawa correspondence of degree two for arbitrary level," J. Ramanujan Math. Soc. 17-3 (2002) 149-160.

[56] H. Aoki, "A note on additive lifts of Jacobi forms with levels" (under preparation).

[57] H. Maaß, "Über ein Analogon zur Vermutung yon Saito-Kurokawa," Inventiones. Math. 601980 85-104. 